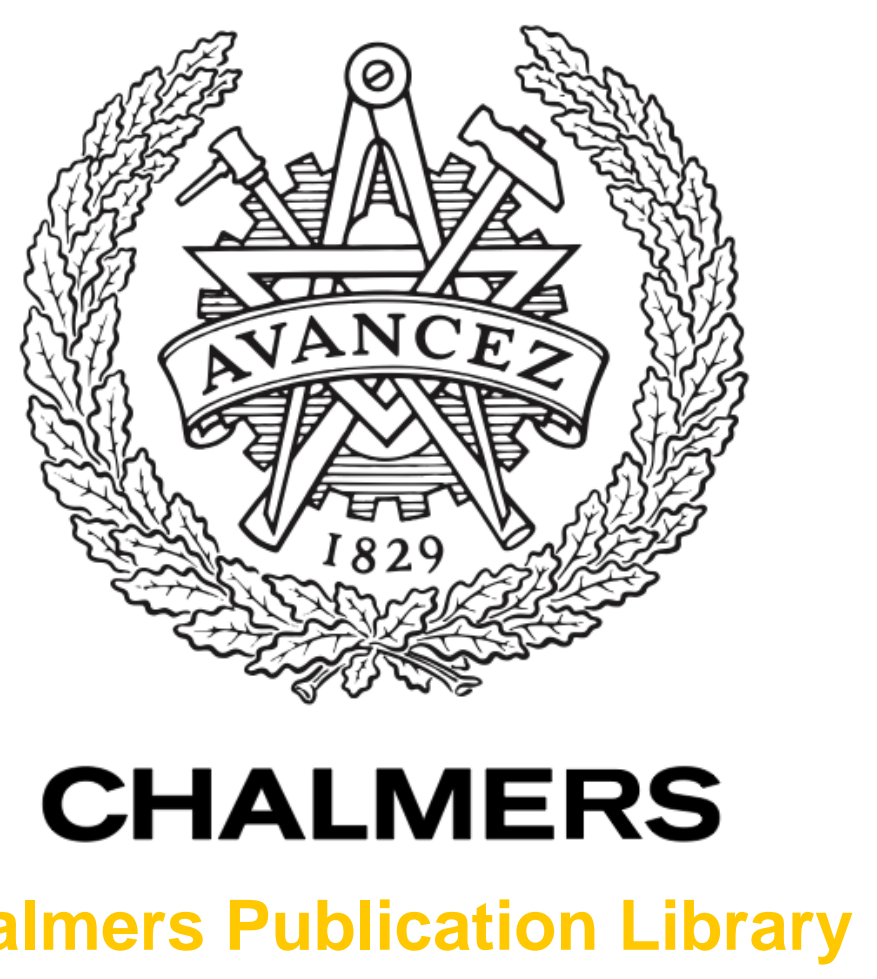

Challmers Publication Library

\title{
Linear Polarization of Class I Methanol Masers in Massive Star-forming Regions
}

This document has been downloaded from Chalmers Publication Library (CPL). It is the author's version of a work that was accepted for publication in:

Astrophysical Journal Supplement Series (ISSN: 0067-0049)

Citation for the published paper:

Kang, J. ; Byun, D. ; Kim, K. et al. (2016) "Linear Polarization of Class I Methanol Masers in Massive Star-forming Regions". Astrophysical Journal Supplement Series, vol. 227(2), pp. 18.

Downloaded from: http://publications.lib.chalmers.se/publication/246349

Notice: Changes introduced as a result of publishing processes such as copy-editing and formatting may not be reflected in this document. For a definitive version of this work, please refer to the published source. Please note that access to the published version might require a subscription. 


\title{
LINEAR POLARIZATION OF CLASS I METHANOL MASERS IN MASSIVE STAR-FORMING REGIONS
}

\author{
Ji-Hyun Kang ${ }^{1}$, Do-Young Byun ${ }^{1}$, KeE-TAe Kim ${ }^{1}$, Jongsoo Kim ${ }^{1,3}$, A-Ran Lyo ${ }^{1}$, And W. H. T. Vlemmings ${ }^{2}$ \\ ${ }^{1}$ Korea Astronomy and Space Science Institute, 776 Daedeokdae-ro, Yuseong-gu, Daejeon 34055, Republic Of Korea; jkang@kasi.re.kr \\ 2 Department of Earth and Space Sciences, Chalmers University of Technology, Onsala Space Observatory, SE-439 92 Onsala, Sweden \\ Received 2016 June 27; revised 2016 September 26; accepted 2016 October 18; published 2016 December 2
}

\begin{abstract}
Class I methanol masers are found to be good tracers of the interaction between outflows from massive young stellar objects with their surrounding media. Although polarization observations of Class II methanol masers have been able to provide information about magnetic fields close to the central (proto)stars, polarization observations of Class I methanol masers are rare, especially at 44 and $95 \mathrm{GHz}$. We present the results of linear polarization observations of 39 Class I methanol maser sources at 44 and $95 \mathrm{GHz}$. These two lines are observed simultaneously with one of the $21 \mathrm{~m}$ Korean VLBI Network telescopes in single-dish mode. Approximately $60 \%$ of the observed sources have fractional polarizations of a few percent in at least one transition. This is the first reported detection of linear polarization of the $44 \mathrm{GHz}$ methanol maser. The two maser transitions show similar polarization properties, indicating that they trace similar magnetic environments, although the fraction of the linear polarization is slightly higher at $95 \mathrm{GHz}$. We discuss the association between the directions of polarization angles and outflows. We also discuss some targets having different polarization properties at both lines, including DR21(OH) and G82.58+0.20, which show the $90^{\circ}$ polarization angle flip at $44 \mathrm{GHz}$.
\end{abstract}

Key words: ISM: magnetic fields - masers - polarization - stars: massive - stars: protostars - surveys

\section{INTRODUCTION}

Even though magnetic fields are thought to play an important role in regulating the formation of stars, there are still many questions regarding their exact shape, strength, and effect (e.g., Crutcher 2012). For example, statistical research on the field alignment in different scales in star-forming cores, i.e., from a parsec-scale cloud down to a $\sim 1000$ au scale envelope, has recently been performed with a limited number of samples, and the correlation between the field orientation and the outflow direction is still under debate (Chapman et al. 2013; Hull et al. 2013, 2014; Surcis et al. 2013; Zhang et al. 2014). To reveal the field properties in star-forming regions at various scales and densities, observations of dust continuum polarization have been used to study the envelopes at scales of several thousand astronomical units (Koch et al. 2010; Tang et al. 2013; Hull et al. 2014; Stephens et al. 2014). On the other hand, masers $\left(\mathrm{OH}, \mathrm{H}_{2} \mathrm{O}, \mathrm{SiO}\right.$, and $\left.\mathrm{CH}_{3} \mathrm{OH}\right)$ are the only tracers that can be used to study the physical conditions of dense regions $\left(n_{\mathrm{H}}=10^{5-11} \mathrm{~cm}^{-3}\right)$, close to protostellar disks or outflows, that are embedded in thick dust envelopes. For a long time, masers were thought to trace the isolated pockets of a compressed field rather than the field of an ambient medium. However, some recent studies of $1.6 \mathrm{GHz}$ hydroxyl and 6.7 $\mathrm{GHz}$ methanol masers showed that they possibly probe the large-scale magnetic field (e.g., Fish \& Reid 2006; Vlemmings et al. 2010; Surcis et al. 2012).

Class I methanol masers, including 44 and $95 \mathrm{GHz}$ transitions, are known to trace the regions where the outflow of a massive young stellar object is interacting with the surrounding medium, while Class II methanol masers are tightly correlated with the central (proto)stars (Plambeck \& Menten 1990; Menten 1991; Kurtz et al. 2004; Gómez-Ruiz et al. 2016). Water masers are also related to outflows, but they appear to arise from shocked regions closer to the central object

\footnotetext{
3 Korea University of Science and Technology, 217 Gajeong-ro, Yuseong-gu, Daejeon 34113, Republic of Korea.
}

than Class I methanol masers (e.g., Kurtz et al. 2004). The polarimetric observations of Class II methanol masers have been increased during the last decade (e.g., Vlemmings 2012) and have been able to provide information about the magnetic fields near the central objects. However, the polarization studies of Class I methanol masers have been very limited. For example, the detection of linear polarizations at $95 \mathrm{GHz}$ has been reported only for two sources (Wiesemeyer et al. 2004). No linear polarization has been reported for $44 \mathrm{GHz}$ methanol maser sources, whereas circular polarization at $44 \mathrm{GHz}$, detected with the Very Large Array (VLA), has been presented only for one object, OMC2 (Sarma \& Momjian 2011). Therefore, further linear and circular polarization observations of the Class I methanol masers need to be performed.

In this paper, we report on single-dish measurements of linear polarization toward 44 and $95 \mathrm{GHz}$ methanol maser sources. Interpreting the single-dish polarization results is not straightforward. Many maser features with different polarization properties within a large single-dish beam are blended in a spectrum. Furthermore, the fractional polarization is affected by maser saturation as well as by the angle between the maser propagation direction and the yet unknown magnetic field direction. However, single-dish polarization observations still contribute significantly to improving our understanding of the masers and the magnetic field in the maser region. Specifically, polarization results for large source samples provide a statistical view on the polarization properties of 44 and $95 \mathrm{GHz}$ methanol masers. As the 44 and $95 \mathrm{GHz}$ masers are believed to trace similar regions (e.g., Val'tts et al. 2000; Kang et al. 2015), their polarization angles are expected to be similar. By observing the two simultaneously, we can examine whether this is the case. Additionally, Wiesemeyer et al. (2004) have detected a high fraction of linear polarizations $(>30 \%)$ for some of their targets, which requires specific conditions of anisotropic pumping or loss mechanisms for high $J$ transitions, where $J$ is a rotational quantum number. With further 
observations, we can verify whether these conditions are indeed regularly achieved.

This paper is structured as follows. Section 2 describes source selection criteria, observation, and reduction methods. Section 3 presents the statistics of polarization results. We discuss the implication of the measured polarization properties, the similarity or difference of the polarization properties of 44 and $95 \mathrm{GHz}$ masers, and the possible association of maser polarization angles with larger-scale fields, in Section 4. We discuss some individual targets with some unique polarization properties in Section 5. We summarize our study in Section 6.

\section{OBSERVATIONS AND DATA REDUCTION}

\subsection{Target Selection and Coordinate Refinement}

We observed 39 sources, which are listed in Table 1. We selected 36 bright Class I methanol maser sources with peak flux densities $\gtrsim 50 \mathrm{Jy}$ from the Korean VLBI Network (KVN) $44 \mathrm{GHz}$ methanol maser survey (Kim et al. 2012). We also observed three weaker sources (S231, W51Met2, W75S(3)), for which linear polarizations were detected by Wiesemeyer et al. (2004) in Class I methanol maser transitions but were not included in the KVN $44 \mathrm{GHz}$ methanol maser survey.

Except for some targets observed with the VLA at $44 \mathrm{GHz}$ (Kogan \& Slysh 1998; Kurtz et al. 2004), many of the coordinates used in the KVN $44 \mathrm{GHz}$ methanol maser survey are the positions of central (proto)stars. Class I methanol masers are generally located offset from the central (proto)stars (e.g., Kurtz et al. 2004). The peaks of such Class I methanol masers may have offsets from the central objects, which would affect the measured peak flux density and polarization properties. A mispointing of $10^{\prime \prime}$ at $95 \mathrm{GHz}\left(15^{\prime \prime}\right.$ at $\left.44 \mathrm{GHz}\right)$ can produce $1 \%$ of artificial polarization (see Section 2.2). To solve this issue, we carefully adjusted target coordinates. For the source with coordinates of a central object, we made a 9-point grid map. After the interpolation of the observed maser intensities between the 9 points, we defined new coordinates if the offsets were larger than $\sim 10^{\prime \prime}$. The sources included in the KVN VLBI fring survey (K. T. Kim et al. 2016, in preparation) have refined positions for the $44 \mathrm{GHz}$ methanol masers. The coordinates of those sources were adopted from the fring survey. In addition, we observed five points around the source with $30^{\prime \prime}$ spacing before each polarization measurement. After similar interpolation of intensities between the 5 points, we subsequently calculated the az/el offsets of the $44 \mathrm{Ghz}$ maser peak from the original coordinates. Then we carried out the polarization observations at the interpolated peak positions. We note that L379 and NGC7538 has large offsets $>24^{\prime \prime}$, and we determined their coordinates based on the 9-point grid maps. The coordinates used for the polarization measurements and their references are described in Table 1.

\subsection{Observations and Calibration}

We observed the 39 sources at 44 and $95 \mathrm{GHz}$ simultaneously in full polarization spectral mode. The observed lines are the Class I methanol $7_{0}-6_{1} A+(44.06943 \mathrm{GHz})$ and $8_{0}-7_{1} A+(95.169463 \mathrm{GHz})$ maser transitions. The observations were conducted using the KVN $21 \mathrm{~m}$ telescope at the Yonsei station in the single-dish mode from August to December in 2013. The beam sizes are $65^{\prime \prime}$ and $30^{\prime \prime}$ at 44 and $95 \mathrm{GHz}$, respectively (Kim et al. 2011; Lee et al. 2011). A digital filter and a FX type digital spectrometer were used as a backend. The spectrometer can produce auto-power spectra of four input signals and cross-power spectra of two input pairs simultaneously, enabling us to derive full Stokes parameters $(I, Q, U, V)$ from the two polarization pairs (Oh et al. 2011, D. Y. Byun et al. 2016, in preparation). Each spectrum of the spectrometer has 4096 channels. The digital filter and spectrometer were configured to have a bandwidth of $64 \mathrm{MHz}$ for each spectrum, which results in a velocity coverage of $430 \mathrm{~km} \mathrm{~s}^{-1}$ and a velocity resolution of $0.1 \mathrm{~km} \mathrm{~s}^{-1}$ at $44 \mathrm{GHz}$.

We used the Walsh Position Switching mode (Mangum 2000), which repeats several pairs of $6 \mathrm{~s}$ on/off integration after firing cal. For data reduction, we used the python polarization data pipeline module available in KVN. The instrumental cross-talk and phase offset were corrected using planets (Jupiter, Venus, or Mars) and Crab, which were observed at least once a day as calibrators. The final spectra at 44 and $95 \mathrm{GHz}$ were reduced to have the same velocity resolution of $0.2 \mathrm{~km} \mathrm{~s}^{-1}$. The typical rms levels $(1 \sigma)$ of the observed spectra are $0.5 \mathrm{Jy}$ and $1.2 \mathrm{Jy}$ at 44 and $95 \mathrm{GHz}$, respectively.

The polarized intensity (PI) given here is $\left(Q^{2}+U^{2}\right)^{1 / 2}$, and the given error is the standard deviation of the measurement sets. The detection criterion is that $\mathrm{PI}>3 \sigma$. The measured polarization angle was derived by $\chi=\frac{1}{2} \arctan \left(\frac{U}{Q}\right)+152^{\circ}$. Here the latter term is the absolute polarization angle of Crab, which is known to be nearly constant near the brightest region from millimeter to X-ray wavelengths (Aumont et al. 2010).

Details on the single-dish polarization observation and calibration using KVN will be presented in a separate paper (D. Y. Byun et al. 2016, in preparation). The procedures of data reduction in the KVN polarization system are also briefly explained in Kang et al. (2015). We also briefly describe the process here. The KVN digital spectrometer backend for the polarization observations produces two single-polarization spectra and one complex cross-polarization spectrum, which are associated with the four Stokes parameters, $I, Q, U$, and $V$, as Sault et al. (1996) mentioned. To estimate the leakage, so called combined D-term, from the total intensity to the crosspolarization spectrum, we used unpolarized planets, for which $Q=U=V=0$. The D-term measured by Jupiter were about $4 \%$ and $8 \%$ at 44 and $95 \mathrm{GHz}$, respectively. To evaluate the errors of the D-term, we observed planets multiple times in a day, and calibrated the unpolarized planets either using another planet or using the same planet observed with some time interval in a day. For the Jupiter-Jupiter, Venus-Venus, or Jupiter-Venus pairs, the measured polarization fractions were less than $0.4 \%$ and $2 \%$ at 44 and $95 \mathrm{GHz}$, respectively. These values would be the upper limits for the errors of the D-term because they include the thermal noise of measurements. Thus, the sources with polarization fractions above those values can be treated as real, not artificial.

The polarization angles of targets were corrected based on the polarization angle of Crab observed on the same day. We have found that the polarization angles of Crab stayed constant within $4^{\circ}$ at 44 and $95 \mathrm{GHz}$ when they were measured on the same day.

The leakage variation due to the offset of the source from the beam center was tested in the KVN system using unpolarized sources, 3C 84, Jupiter, Venus, and Mars at $43 \mathrm{GHz}$ at offsets from $0^{\prime \prime}$ to $30^{\prime \prime}$ with $5^{\prime \prime}$ or $10^{\prime \prime}$ intervals. The results showed that the fractions of artificial polarization due to the pointing offsets 
Table 1

Source Summary

\begin{tabular}{|c|c|c|c|c|c|c|c|c|c|c|}
\hline \multirow[b]{2}{*}{$\begin{array}{l}\text { Source } \\
\text { Name }\end{array}$} & \multirow[b]{2}{*}{$\begin{array}{c}\alpha_{2000} \\
(\mathrm{~h}: \mathrm{m}: \mathrm{s})\end{array}$} & \multirow[b]{2}{*}{$\begin{array}{c}\delta_{2000} \\
\left({ }^{\circ}:{ }^{\prime}: "\right)\end{array}$} & \multicolumn{2}{|c|}{$F \nu$} & \multicolumn{2}{|c|}{$v_{\mathrm{LSR}}$} & \multicolumn{2}{|c|}{$\sigma$} & \multirow[b]{2}{*}{$\begin{array}{c}\text { Pol } \\
44 / 95\end{array}$} & \multirow[b]{2}{*}{ Ref } \\
\hline & & & \multicolumn{2}{|c|}{ (Jy) } & \multicolumn{2}{|c|}{$\left(\mathrm{km} \mathrm{s}^{-1}\right)$} & \multicolumn{2}{|c|}{ (Jy) } & & \\
\hline OMC2 & $05: 35: 27.124$ & $-05: 09: 52.48$ & 210 & 290 & +11.1 & +11.1 & 0.36 & 0.71 & $\mathrm{Y} / \mathrm{Y}$ & (1) \\
\hline $\mathrm{S} 231^{\mathrm{a}}$ & $05: 39: 13.060$ & $+35: 45: 51.30$ & 26 & 22 & -16.7 & -16.7 & 0.25 & 0.72 & $\mathrm{~N} / \mathrm{N}$ & (2) \\
\hline S235 & $05: 40: 53.250$ & $+35: 41: 46.90$ & 74 & 60 & -16.4 & -16.4 & 0.31 & 0.83 & $\mathrm{~N} / \mathrm{N}$ & (1) \\
\hline S255N & $06: 12: 53.630$ & $+18: 00: 25.10$ & 260 & 190 & +11.0 & +11.0 & 0.30 & 0.52 & $\mathrm{Y} / \mathrm{Y}$ & (1) \\
\hline NGC2264 & 06:41:08.073 & $+09: 29: 39.99$ & 170 & 120 & +7.2 & +7.2 & 0.33 & 0.85 & $\mathrm{~N} / \mathrm{Y}$ & (1) \\
\hline G357.96-0.16 & $17: 41: 20.140$ & $-30: 45: 14.40$ & 66 & 47 & -5.0 & -5.0 & 0.42 & 0.69 & $\mathrm{Y} / \mathrm{Y}$ & (3) \\
\hline G359.61-0.24 & $17: 45: 39.080$ & $-29: 23: 29.00$ & 99 & 53 & +19.3 & +19.3 & 0.47 & 1.21 & $\mathrm{Y} / \mathrm{N}$ & (1) \\
\hline G0.67-0.02 & $17: 47: 19.230$ & $-28: 22: 14.50$ & 33 & 30 & +69.2 & +65.5 & 1.94 & 4.38 & $\mathrm{~N} / \mathrm{N}$ & (1) \\
\hline IRAS18018-2426 ${ }^{\mathrm{b}}$ & 18:04:53.010 & $-24: 26: 40.50$ & 690 & 260 & +10.9 & +10.8 & 0.52 & 0.88 & $\mathrm{Y} / \mathrm{Y}$ & (3) \\
\hline G10.34-0.14 & 18:09:00.000 & $-20: 03: 35.00$ & 110 & 78 & +14.6 & +14.6 & 0.39 & 0.66 & $\mathrm{Y} / \mathrm{Y}$ & (3) \\
\hline G10.32-0.26 & $18: 09: 22.860$ & $-20: 08: 05.80$ & 170 & 69 & +32.4 & +32.5 & 0.50 & 0.86 & $\mathrm{Y} / \mathrm{N}$ & (3) \\
\hline G10.62-0.38 & $18: 10: 29.070$ & $-19: 55: 48.20$ & 100 & 28 & -6.8 & -6.7 & 0.60 & 2.14 & $\mathrm{~N} / \mathrm{N}$ & (1) \\
\hline G11.92-0.61 & $18: 13: 58.100$ & $-18: 54: 31.00$ & 67 & 30 & +35.2 & +35.2 & 0.60 & 1.52 & $\mathrm{~N} / \mathrm{N}$ & (4) \\
\hline W33MET & $18: 14: 11.183$ & $-17: 56: 00.00$ & 54 & 36 & +32.8 & +32.8 & 0.81 & 1.81 & $\mathrm{~N} / \mathrm{Y}$ & (4) \\
\hline G13.66-0.60 & $18: 17: 24.080$ & $-17: 22: 14.10$ & 65 & 47 & +48.3 & +48.4 & 0.73 & 1.88 & $\mathrm{~N} / \mathrm{N}$ & (1) \\
\hline G18.34+1.78SW & $18: 17: 49.950$ & $-12: 08: 06.48$ & 620 & 390 & +30.4 & +30.3 & 0.43 & 0.77 & $\mathrm{Y} / \mathrm{Y}$ & (3) \\
\hline G14.33-0.63 & $18: 18: 54.200$ & $-16: 48: 00.50$ & 170 & 100 & +23.3 & +23.4 & 0.83 & 2.16 & $\mathrm{~N} / \mathrm{N}$ & (4) \\
\hline GGD27 & $18: 19: 12.450$ & $-20: 47: 24.80$ & 130 & 58 & +13.2 & +13.1 & 0.60 & 1.56 & $\mathrm{Y} / \mathrm{Y}$ & (1) \\
\hline G19.36-0.03 & $18: 26: 25.800$ & $-12: 03: 57.00$ & 130 & 79 & +26.5 & +26.2 & 0.44 & 0.84 & $\mathrm{Y} / \mathrm{N}$ & (1) \\
\hline L379 & $18: 29: 23.307$ & $-15: 15: 29.92$ & 140 & 77 & +17.6 & +17.6 & 0.51 & 0.92 & $\mathrm{Y} / \mathrm{Y}$ & (4) \\
\hline $\mathrm{G} 25.65+1.04$ & $18: 34: 20.910$ & $-05: 59: 40.50$ & 63 & 60 & +41.6 & +41.6 & 0.46 & 1.09 & $\mathrm{~N} / \mathrm{N}$ & (4) \\
\hline G23.43-0.18 & $18: 34: 39.270$ & $-08: 31: 39.00$ & 71 & 32 & +101.3 & +102.3 & 0.32 & 1.91 & $\mathrm{Y} / \mathrm{Y}$ & (3) \\
\hline G25.82-0.17 & 18:39:03.630 & $-06: 24: 09.50$ & 83 & 81 & +90.4 & +90.2 & 0.38 & 2.48 & $\mathrm{~N} / \mathrm{N}$ & (1) \\
\hline G27.36-0.16 & $18: 41: 50.980$ & $-05: 01: 28.00$ & 180 & 100 & +94.0 & +94.1 & 0.36 & 0.85 & $\mathrm{Y} / \mathrm{Y}$ & (4) \\
\hline G28.37-0.07MM1 & $18: 42: 52.100$ & $-03: 59: 45.00$ & 61 & 32 & +76.4 & +76.5 & 0.48 & 0.80 & $\mathrm{~N} / \mathrm{N}$ & (3) \\
\hline G28.39+0.08 & $18: 42: 54.500$ & $-04: 00: 04.00$ & 49 & 29 & +79.4 & +79.4 & 0.39 & 0.64 & $\mathrm{~N} / \mathrm{N}$ & (1) \\
\hline G29.91-0.03 & 18:46:05.370 & $-02: 42: 17.10$ & 410 & 220 & +98.2 & +98.2 & 0.42 & 0.77 & $\mathrm{Y} / \mathrm{Y}$ & (4) \\
\hline G30.82-0.05 & $18: 47: 46.840$ & $-01: 54: 14.00$ & 46 & 32 & +96.9 & +96.6 & 0.39 & 1.26 & $\mathrm{Y} / \mathrm{N}$ & (4) \\
\hline G40.25-0.19 & $19: 05: 41.440$ & $+06: 26: 08.00$ & 130 & 120 & +72.7 & +72.7 & 0.33 & 0.58 & $\mathrm{Y} / \mathrm{Y}$ & (4) \\
\hline G49.49-0.39 & $19: 23: 43.960$ & $+14: 30: 31.00$ & 97 & 45 & +49.1 & +55.7 & 0.88 & 2.31 & $\mathrm{Y} / \mathrm{Y}$ & (1) \\
\hline $\mathrm{W} 1 \mathrm{MET}^{\mathrm{a}}$ & $19: 23: 46.500$ & $+14: 29: 41.00$ & 32 & 25 & +56.6 & +56.6 & 0.37 & 0.84 & $\mathrm{~N} / \mathrm{N}$ & (2) \\
\hline G59.79+0.63 & 19:41:03.100 & $+24: 01: 15.00$ & 110 & 67 & +30.8 & +30.8 & 0.22 & 0.67 & $\mathrm{~N} / \mathrm{N}$ & (1) \\
\hline $\mathrm{W} 75 \mathrm{~N}$ & $20: 38: 37.190$ & $+42: 38: 08.00$ & 25 & 13 & +8.8 & +8.9 & 0.62 & 0.97 & $\mathrm{~N} / \mathrm{N}$ & (4) \\
\hline DR21W & 20:38:54.805 & $+42: 19: 22.50$ & 230 & 210 & -2.6 & -2.5 & 0.21 & 0.48 & $\mathrm{Y} / \mathrm{N}$ & (1) \\
\hline $\mathrm{DR} 21(\mathrm{OH})$ & $20: 38: 59.280$ & $+42: 22: 48.70$ & 390 & 310 & -0.1 & +0.0 & 0.24 & 1.35 & $\mathrm{Y} / \mathrm{Y}$ & (1) \\
\hline DR21 & 20:39:01.760 & $+42: 19: 21.10$ & 88 & 61 & -3.7 & -3.8 & 0.37 & 0.76 & $\mathrm{Y} / \mathrm{N}$ & (1) \\
\hline $\mathrm{W} 75 \mathrm{~S}(3)^{\mathrm{a}}$ & 20:39:03.500 & $+42: 26: 00.20$ & 50 & 30 & -5.1 & -5.2 & 0.74 & 1.79 & $\mathrm{~N} / \mathrm{N}$ & (4) \\
\hline $\mathrm{G} 82.58+0.20$ & $20: 43: 28.480$ & $+42: 50: 00.90$ & 240 & 170 & +10.3 & +10.3 & 0.20 & 0.64 & $\mathrm{Y} / \mathrm{Y}$ & (1) \\
\hline NGC7538 & $23: 13: 42.000$ & $+61: 27: 29.70$ & 57 & 20 & -57.4 & -57.3 & 0.26 & 0.75 & $\mathrm{~N} / \mathrm{N}$ & (4) \\
\hline
\end{tabular}

Notes. $F_{\nu}$ is the peak value in the Stokes $I$ spectrum, and $v_{\text {LSR }}$ is the LSR velocity of the peak channel. The rms $(\sigma)$ was measured in the noise channels in the Stokes $I$ spectrum. The $\mathrm{Y}$ or $\mathrm{N}$ in the Pol column indicates whether the linear polarization for each source is detected or not. The last column shows the coordinate references. (1) is the position of the KVN $44 \mathrm{GHz}$ methanol maser survey (Kim et al. 2012). (2) is from Wiesemeyer et al. (2004). (3) is the position adopted from the KVN fring survey (K. T. Kim et al. 2016, in preparation). (4) is the new position determined by the grid mapping in this paper.

a It is not included in the KVN $44 \mathrm{GHz}$ methanol maser survey, but its linear polarization was detected by Wiesemeyer et al. (2004).

${ }^{\mathrm{b}}$ A separate maser source, M8E, is located at 15" from the position of IRAS18018-2426.

are $1 \%$ at $15^{\prime \prime}$ and $2 \%$ at $30^{\prime \prime}$, respectively. The leakage at $86 \mathrm{GHz}$ was also tested with Jupiter and Mars at offsets from $0^{\prime \prime}$ to $15^{\prime \prime}$ with $5^{\prime \prime}$ interval, and $1 \%$ of artificial polarized emission at $10^{\prime \prime}$ offset was measured.

The distortion of the antenna beam pattern with elevation can cause variation of leakage and it adds polarization calibration errors, too. The gain loss due to the distortion of the beam pattern is represented as a gain curve of antenna. The KVN Yonsei telescope has flat gain curves and symmetric beam patterns over the elevation range from $30^{\circ}$ to $60^{\circ}$, where our polarization observations were conducted. The antenna gain changes only $1 \%$ and $3 \%$ at $43 \mathrm{GHz}$ and $86 \mathrm{GHz}$, respectively, over this elevation range. The beam widths and the beam squints are almost constant over the observed elevation range. The antenna beam widths in azimuth and elevation directions differ by less than $2^{\prime \prime}$, and the beam squints are less than 2 ". 5 both in azimuth and elevation at both 43 and $86 \mathrm{GHz}$. Therefore, we expect that the measured D-term is not 

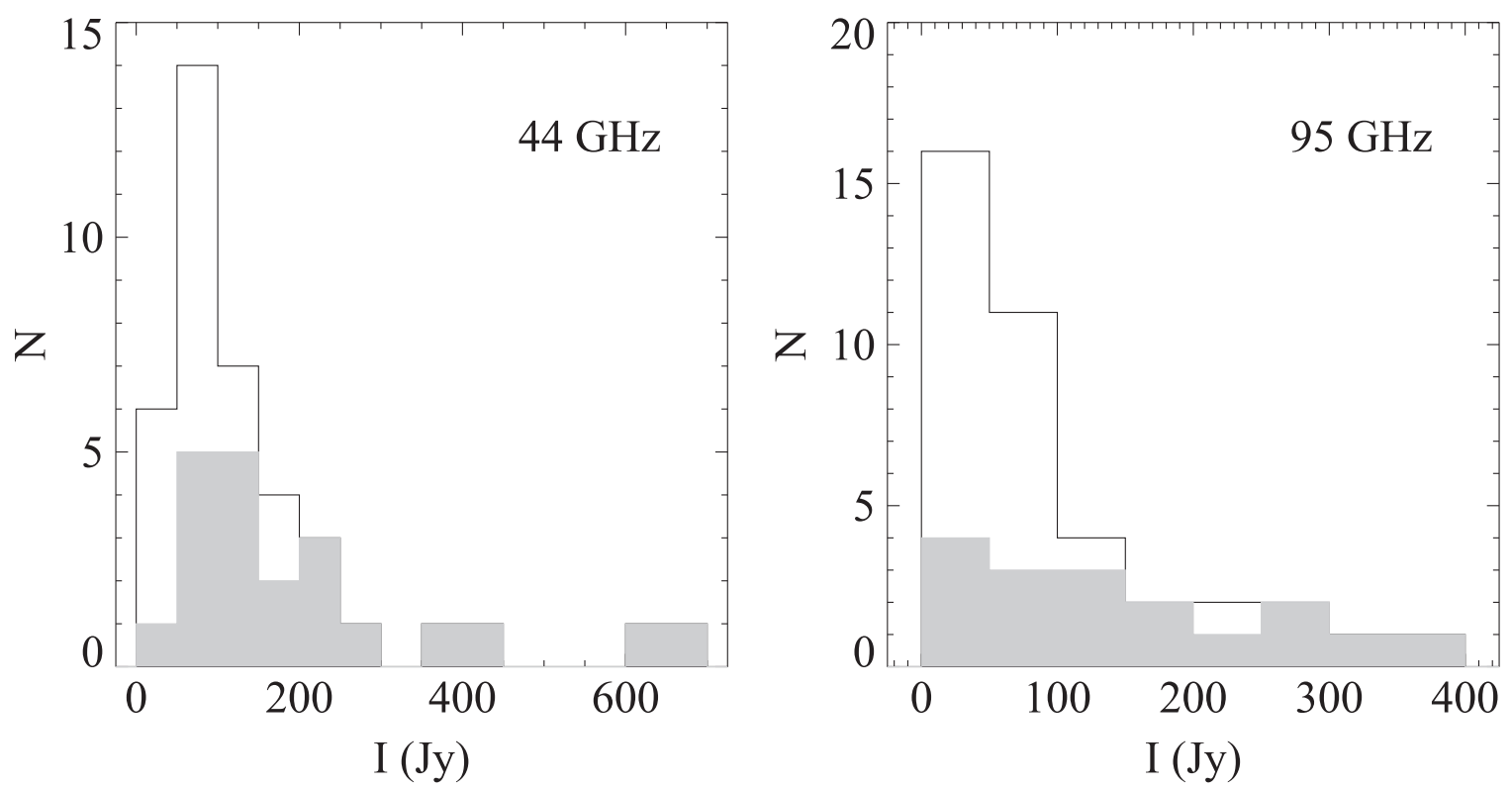

Figure 1. Number density distributions of the total intensity (Stokes $I$ ) of the observed (solid lines) and the polarization-detected sources (gray filled area) at 44 (left) and $95 \mathrm{GHz}$ (right).

Table 2

Linear Polarization Detection Rates

\begin{tabular}{lr}
\hline \hline Group & Number \\
\hline Total Detected & $23(59 \%)$ \\
44 & $21(54 \%)$ \\
95 & $17(44 \%)$ \\
44 and 95 & $15(38 \%)$ \\
44 only & 6 \\
95 only & 2 \\
Total Observed & 39 \\
\hline
\end{tabular}

significantly affected by the antenna beam pattern or beam squint. We note that detailed system performance for the KVN Yonsei telescope is reported in the status report of $\mathrm{KVN}$ (http://radio.kasi.re.kr/kvn/status_report_2014/home.html).

\section{RESULTS}

We detected fractional linear polarization toward $23(59 \%)$ of the 39 Class I methanol maser sources at 44 and/or $95 \mathrm{GHz}$. This detection rate is slightly smaller than that $(71 \%)$ of Wiesemeyer et al. (2004), who found polarization in 10 out of 14 Class I maser sources at 85, 95, and/or $133 \mathrm{GHz}$. Table 2 summarizes the observational results. At 44 and $95 \mathrm{GHz}, 21$ $(54 \%)$ and $17(44 \%)$ sources show linear polarization, respectively. We emphasize that this is the first detection of linear polarization of the $44 \mathrm{GHz}$ methanol masers. Fifteen $(38 \%)$ sources were detected at both frequencies. The rms weighted means of the detected linear polarization fractions are $2.7 \pm 0.3 \%$ and $4.8 \pm 0.1 \%$ at 44 and $95 \mathrm{GHz}$, respectively. Eight sources are detected at only one transition. Among them, NGC2264, which is detected at $95 \mathrm{GHz}$, has $2 \sigma$ possible detection at $44 \mathrm{GHz}$, with about $1 \%$ of fractional polarization. The polarization upper limits of the remaining seven sources are above the measured mean values, implying that the nondetection is due to the sensitivity. It is worth noting that the $95 \mathrm{GHz}$ polarization detections of $\mathrm{OMC} 2$ and $\mathrm{G} 82.58+0.20$ could be affected by the artifacts of the system polarization, because their polarization fractions are the same as or only slightly higher than the upper limit $(2 \%)$ of the artificial polarization of the system at $95 \mathrm{GHz}$.

Figure 1 shows the number density distribution as a function of the total flux for the observed and the polarization-detected sources. The polarization detection rate tends to increase with the total flux at both transitions. Figure 2 presents the distributions of fractional linear polarization, $P_{L}$, at 44 and $95 \mathrm{GHz}$. All sources have $P_{L}<11 \%$ except W33Met $(24.6 \%)$, which has a large error of $6.9 \%(1 \sigma)$. The ranges of polarization fractions are $1.1 \%-9.5 \%$ and $2.0 \%-24.6 \%$ at 44 and $95 \mathrm{GHz}$, respectively. The ranges of upper limits for non-detections are $0.6 \%-8.5 \%$ at $44 \mathrm{GHz}$ and $2 \%-25 \%$ at $95 \mathrm{GHz}$. Figure 3 displays the polarization fraction distributions as a function of total flux. In this figure, the error of $P_{L}$ increases as $I$ decreases, because $P_{L} \propto I^{-1}$ while the observational noise is relatively constant for all sources $(\sim 0.5 \mathrm{Jy}$ at 44 and $\sim 1.2 \mathrm{Jy}$ at $95 \mathrm{GHz})$. The upper limits of polarization non-detected sources are presented with open circles.

Figure 4 presents the spectral profiles of the total flux $(I)$, the PI, the polarization degree $\left(P_{L}\right)$, and the polarization angle measured counterclockwise from north $(\chi)$ of the sources detected at both transitions. The profiles of the total flux and the PI tend to peak at similar velocities, with some exceptions like G40.25-0.19 (Figure 4(l) at $44 \mathrm{GHz}$ ). Some sources, e.g., G18.34+1.78SW (Figure 4(f)), have multiple velocity components with different polarization properties. Figures 5 and 6 show the same parameters for the sources detected only at 44 or $95 \mathrm{GHz}$, respectively.

The measured polarization properties are summarized in Table 3. The presented values of $F_{\nu}, P_{L}, \chi$, and $v_{\text {LSR }}$ are all determined in the channel with the peak PI. The amplitude or the phase of a vector does not have a Gaussian probability distribution unless the signal-to-noise ratio is very large. This can lead to systematic errors in the measured polarizations. According to Wardle \& Kronberg (1974), the best estimates of the true polarization can be found using $R \sim R_{M}{ }^{\prime}\left[1-\left(\sigma^{\prime} / R_{M}{ }^{\prime}\right)^{2}\right]^{1 / 2}$ for $R / \sigma^{\prime}>0.5$, where $R_{M}{ }^{\prime}$ is the observed polarization and $\sigma^{\prime}$ is the 

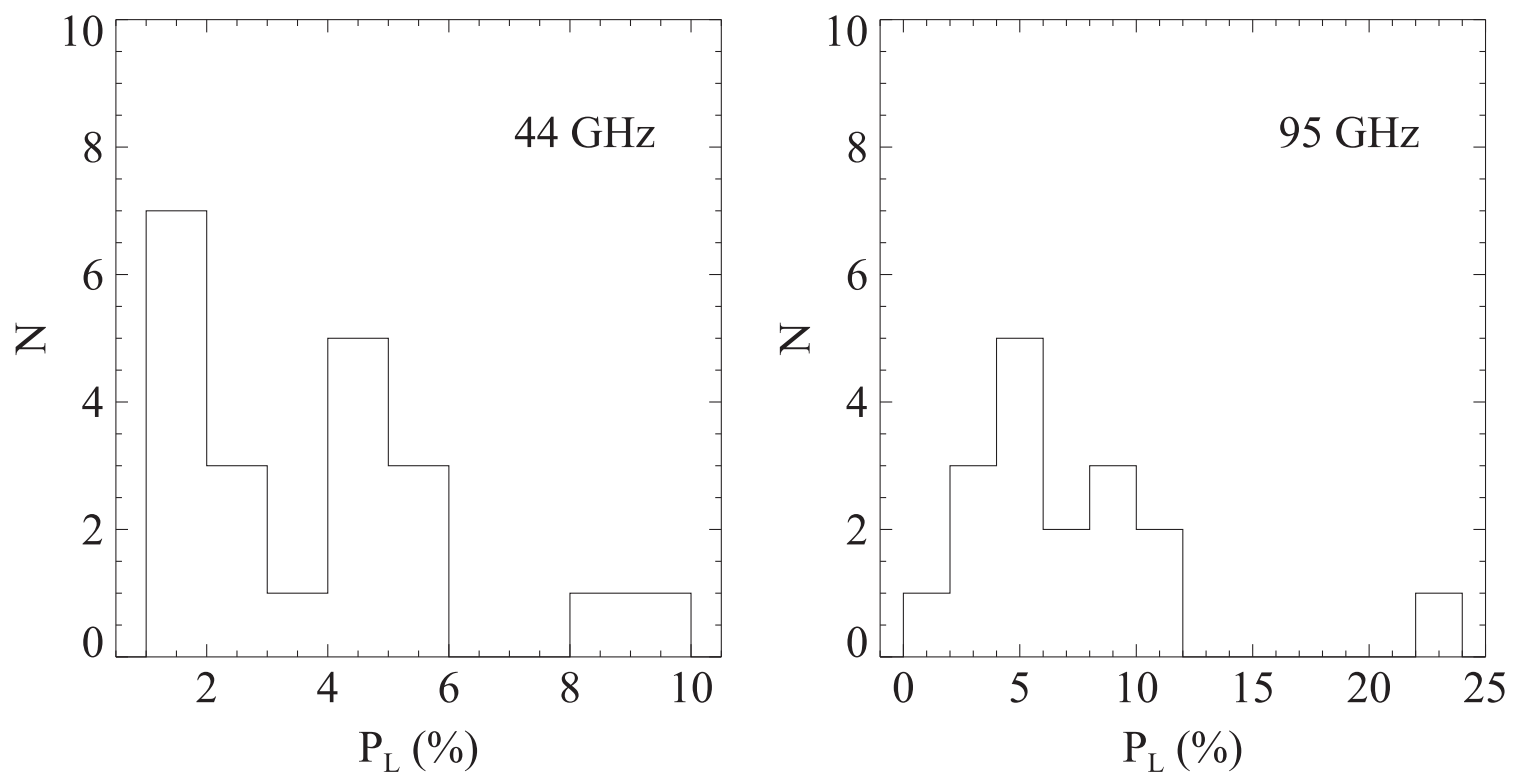

Figure 2. Number density distributions of the polarization fractions at 44 (left) and $95 \mathrm{GHz}$ (right).
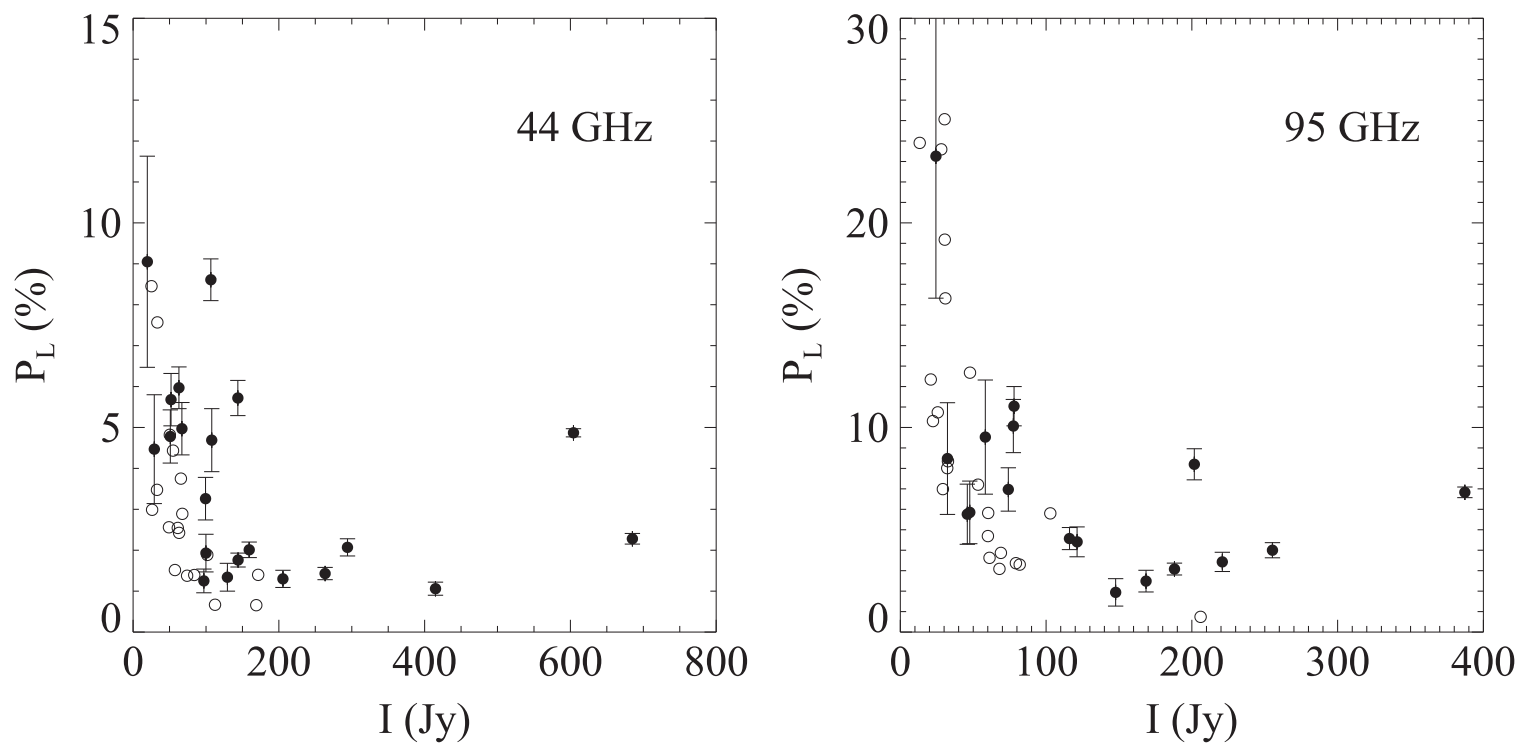

Figure 3. Polarization fraction $P_{L}$ vs. Stokes $I$ at 44 (left) and $95 \mathrm{GHz}$ (right). The filled circles are those detected above $3 \sigma$ errors, while open circles show the upper limits of polarization non-detected sources. Errors of $1 \sigma$ are presented.

observed random noise. Random noise does not produce a bias in $\chi$ but increases the error of $\chi$ by $\sigma_{\chi} \simeq \sigma^{\prime} / R_{M}{ }^{\prime}$ radians. We corrected for these effects in the presented $P_{L}$ and $\chi$. The amount of correction in $P_{L}$ is insignificant in our cases, but $\sigma_{\chi}$ normally exceeds the measurement error. Errors presented are $1 \sigma$.

The polarization properties of the 44 and the $95 \mathrm{GHz}$ methanol masers are observed to be well correlated. In Figure 7, the polarization degrees of the two transitions appear to have a positive linear correlation, although the correlation is not very tight. Their linear Pearson correlation coefficient is $r=0.71$, where $r=0$ and 1 indicate no correlation and a perfect positive linear correlation, respectively. The polarization fractions of the $95 \mathrm{GHz}$ masers tend to be greater than those of the $44 \mathrm{GHz}$ masers. This is consistent with the finding of Wiesemeyer et al. (2004), where the polarization fraction at a higher frequency transition is higher than that at a lower frequency whenever a source is detected at multiple maser transitions. Figure 7 compares the polarization angles of the 44 and the $95 \mathrm{GHz}$ maser transitions as well. Their correlation coefficient is $r=0.92$, indicating that their polarization angles are generally well correlated.

\section{DISCUSSION}

\subsection{The Measured Polarization Properties}

About $40 \%$ of the observed sources did not show any linear polarization in either of the two transitions. Because of the observational limitations such as sensitivity and a large singledish beam depolarizing multiple maser features with different orientations, we cannot exclude the presence of linear polarization even for the non-detected sources. 

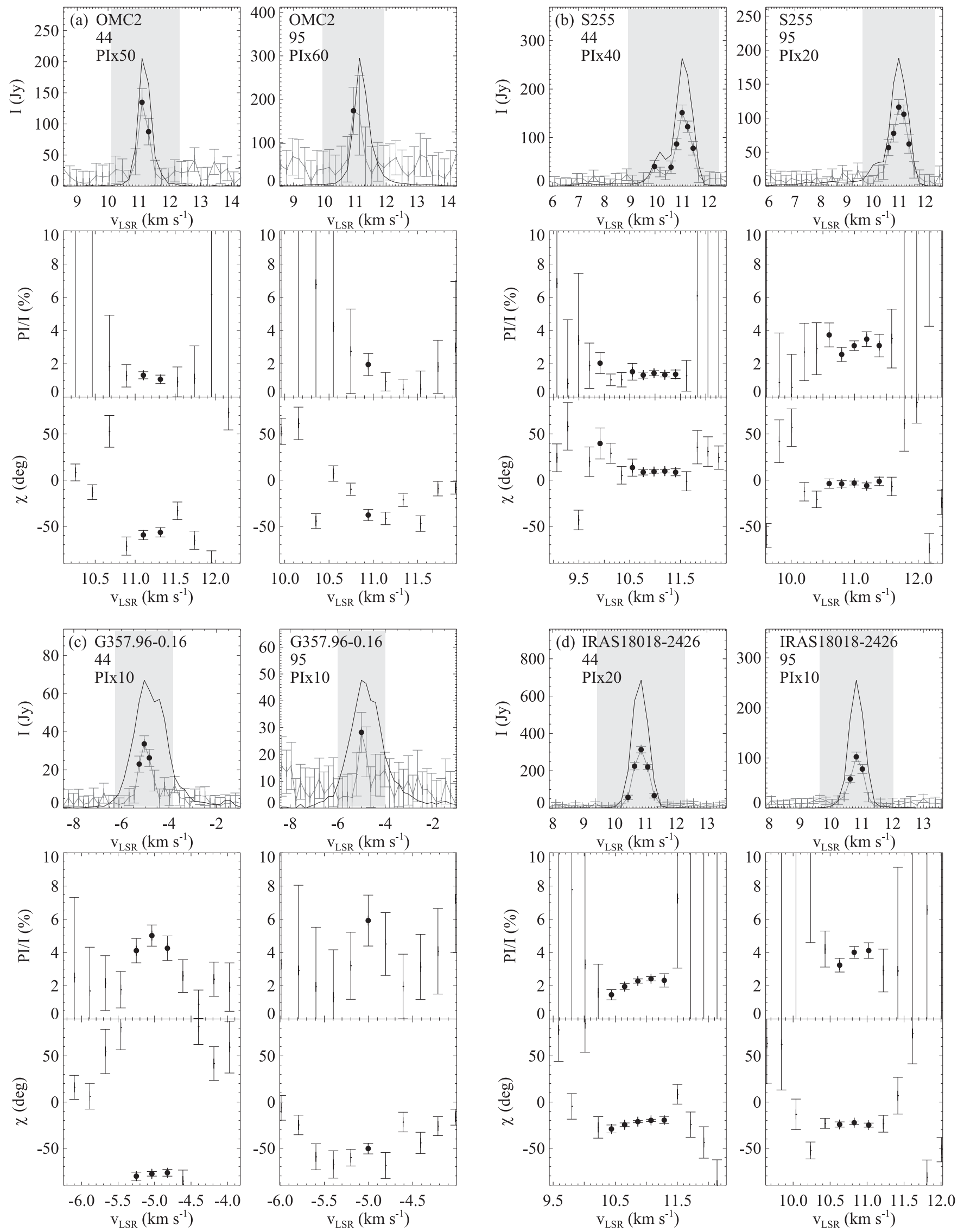

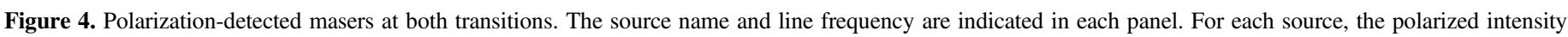

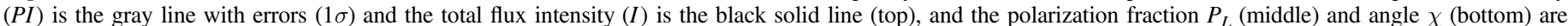

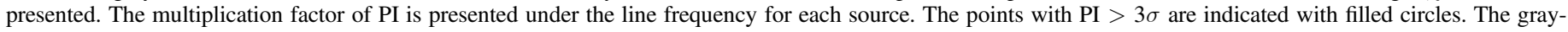
shaded area in the top panel indicates the velocity range for the middle and bottom panels. 

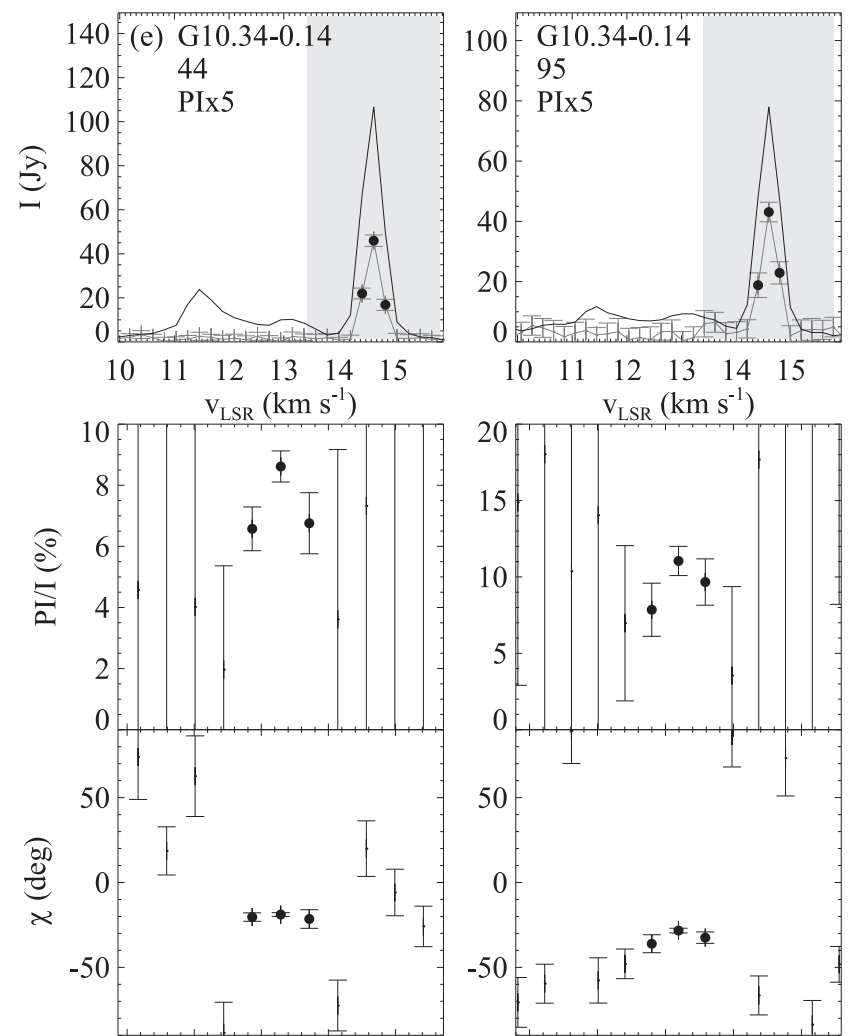

$\begin{array}{lllll}13.5 & 14.0 & 14.5 & 15.0 & 15.5\end{array}$ $\mathrm{v}_{\mathrm{LSR}}\left(\mathrm{km} \mathrm{s}^{-1}\right)$
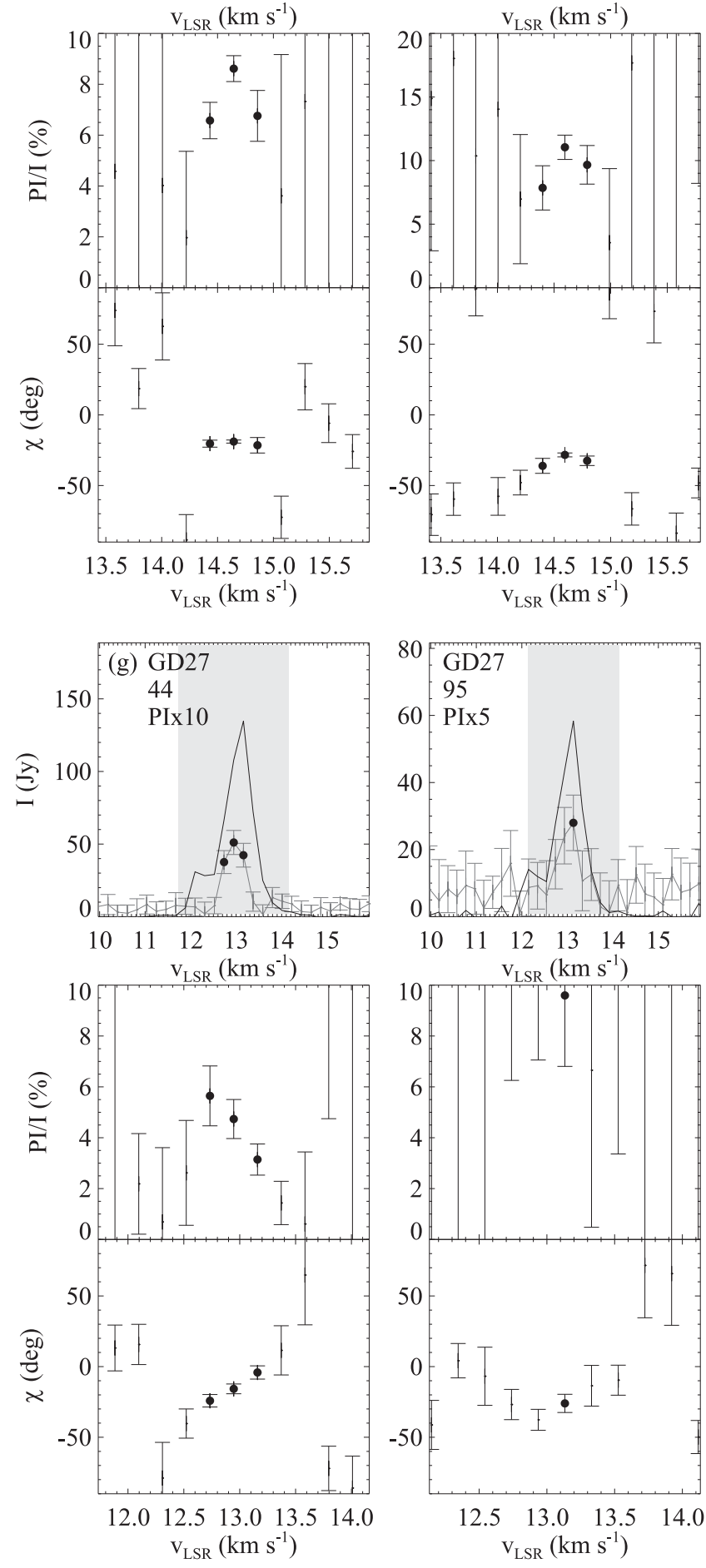

$\begin{array}{lllll}13.5 & 14.0 & 14.5 & 15.0 & 15.5\end{array}$ $\mathrm{V}_{\text {LSR }}\left(\mathrm{km} \mathrm{s}^{-1}\right)$
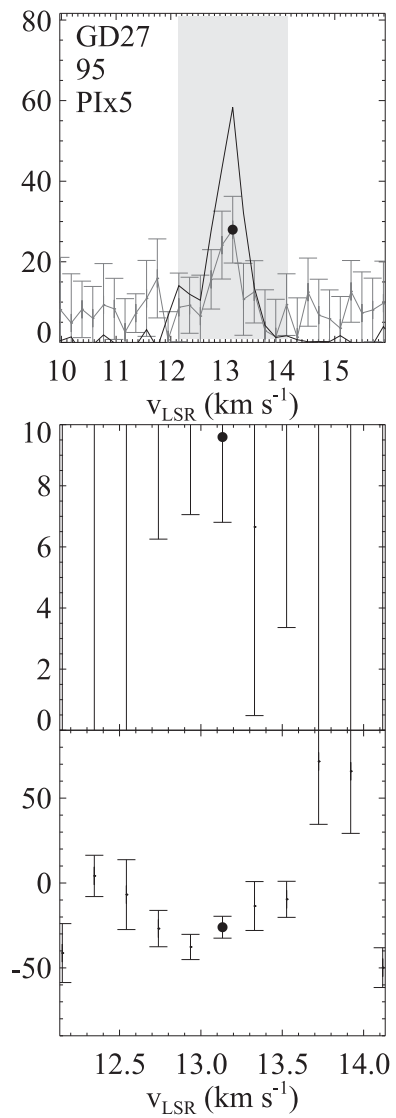

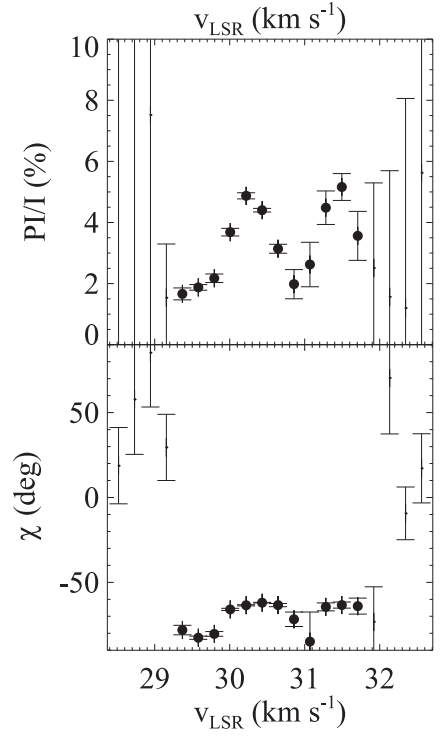

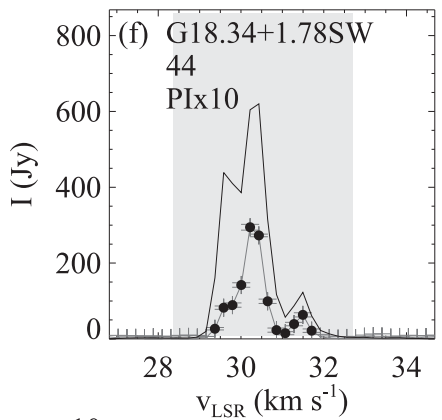
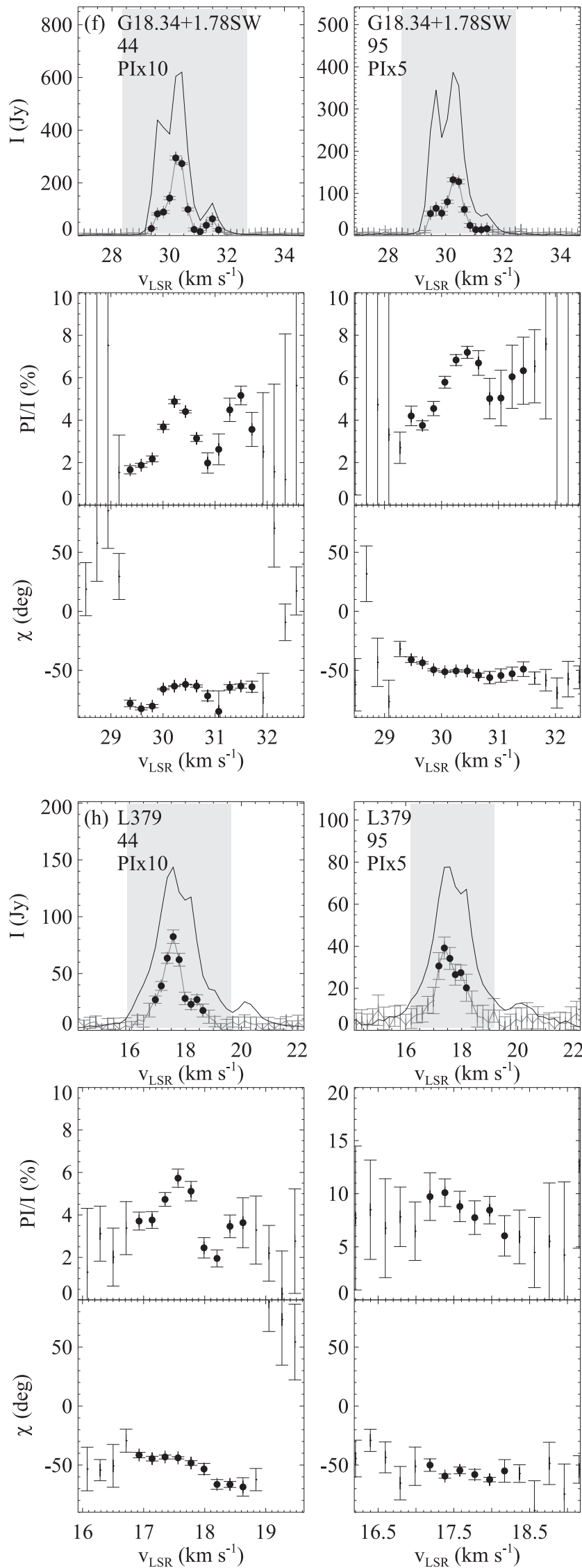

Figure 4. (Continued.) 

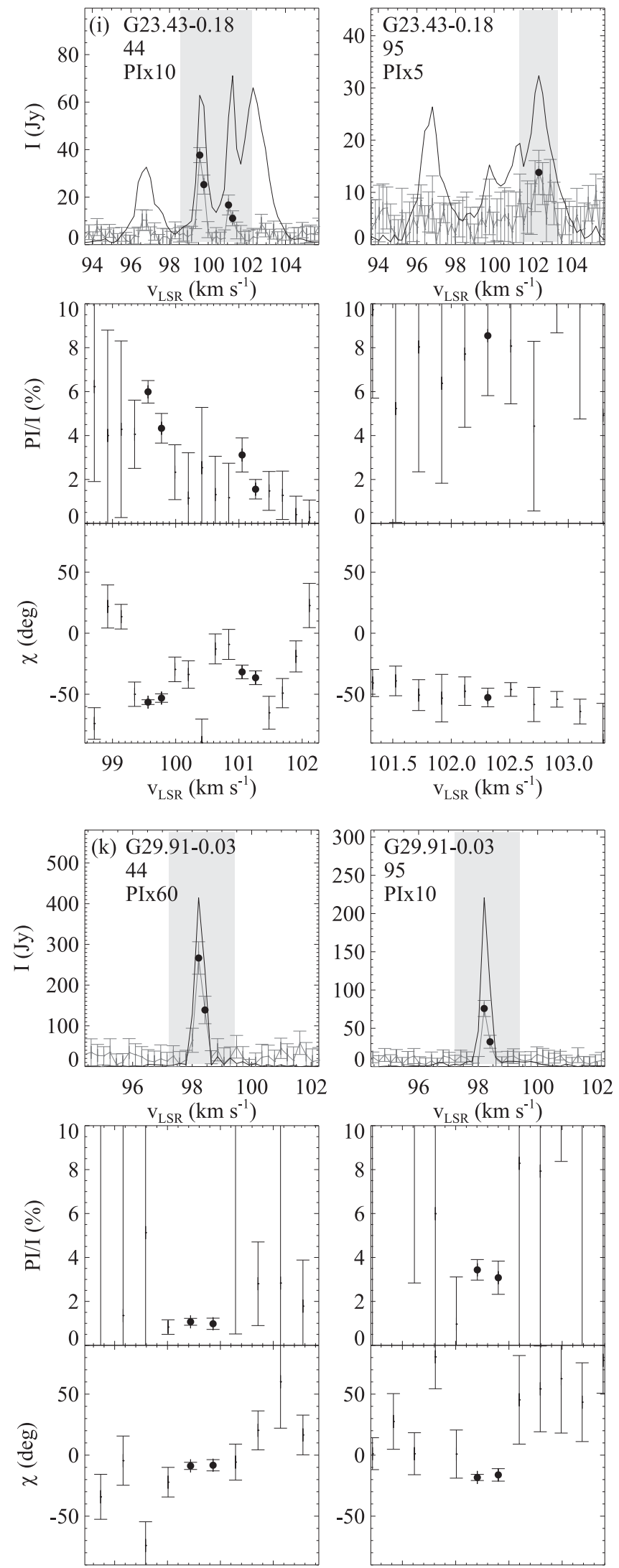

$\begin{array}{llll}97.5 & 98.0 & 98.5 & 99.0\end{array}$

$\mathrm{V}_{\mathrm{LSR}}\left(\mathrm{km} \mathrm{s}^{-1}\right)$

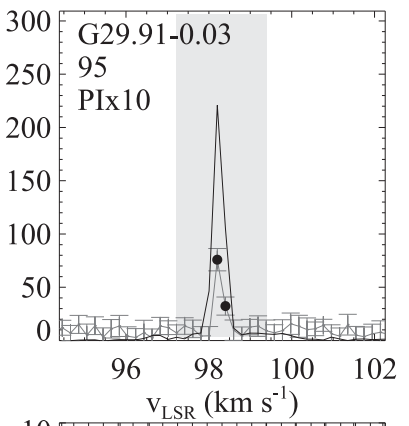

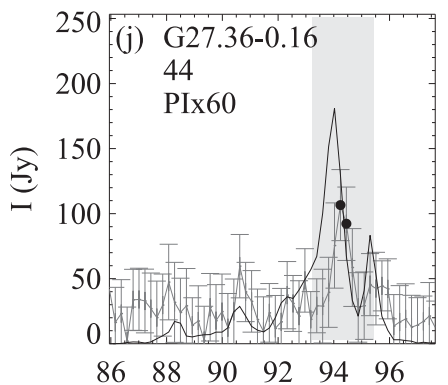
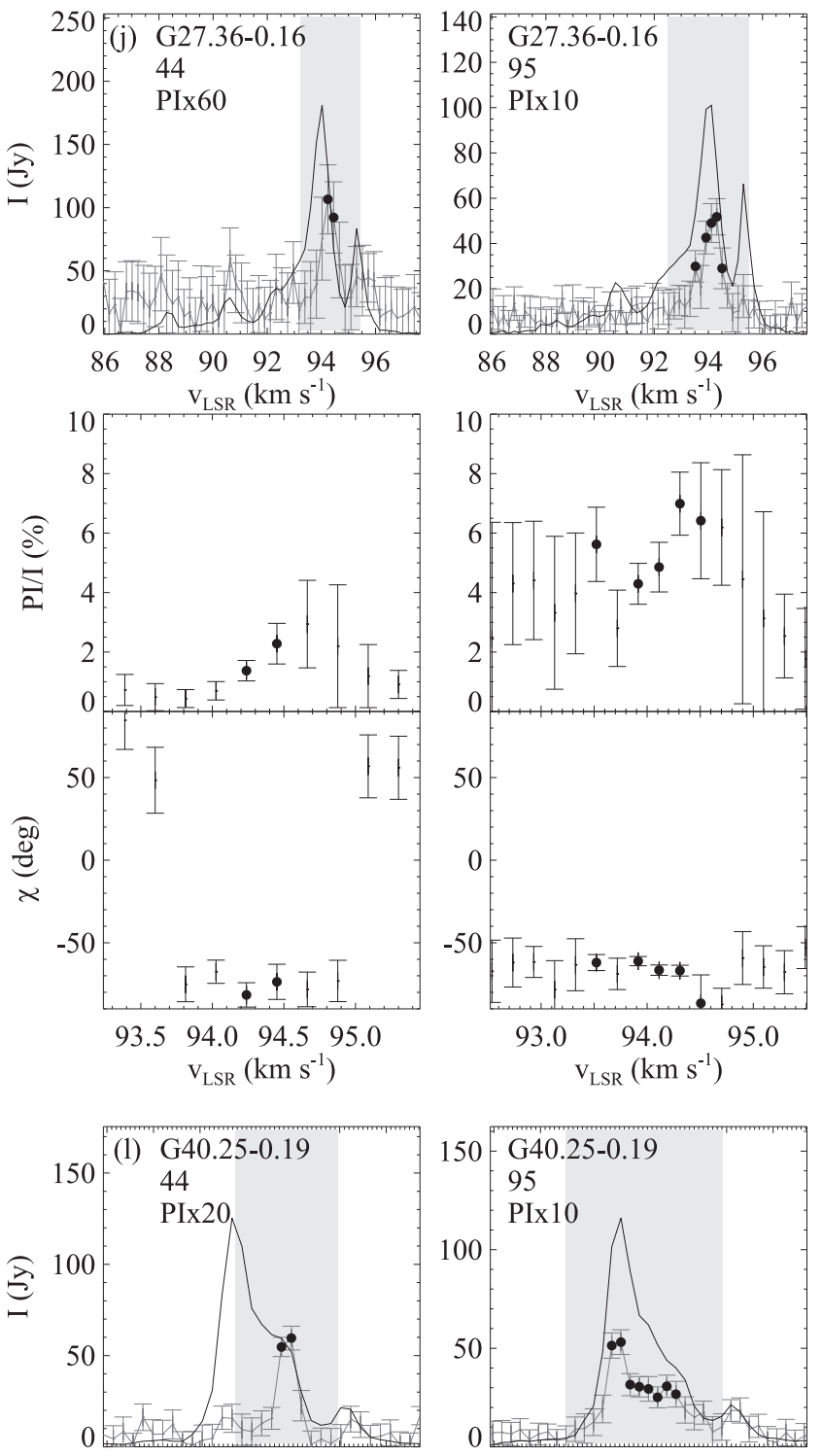

$\begin{array}{lllllll}70 & 71 & 72 & 73 & 74 & 75 & 76\end{array}$
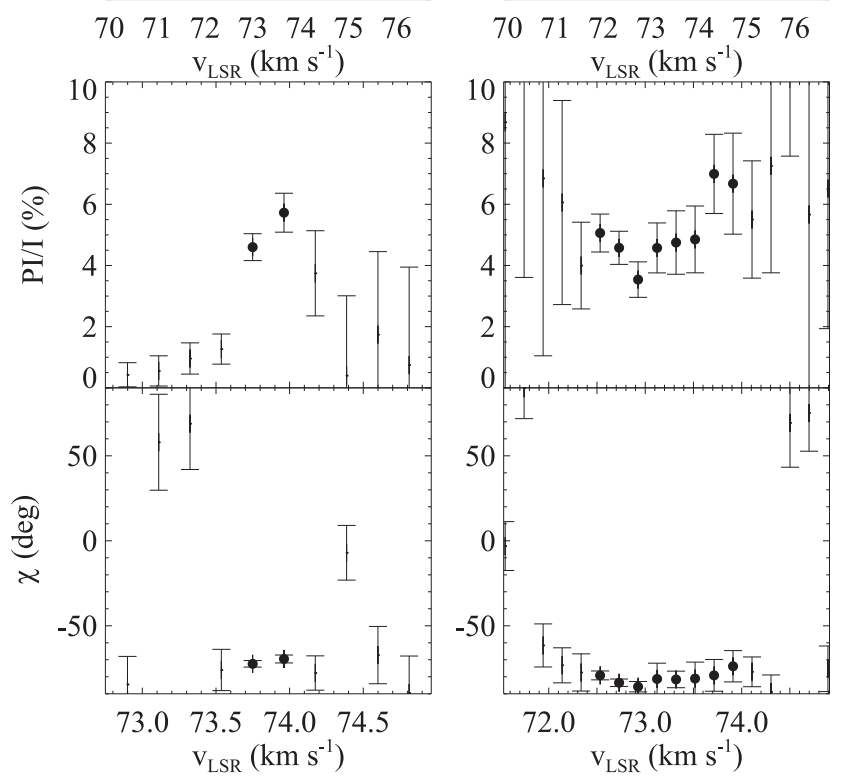

Figure 4. (Continued.) 

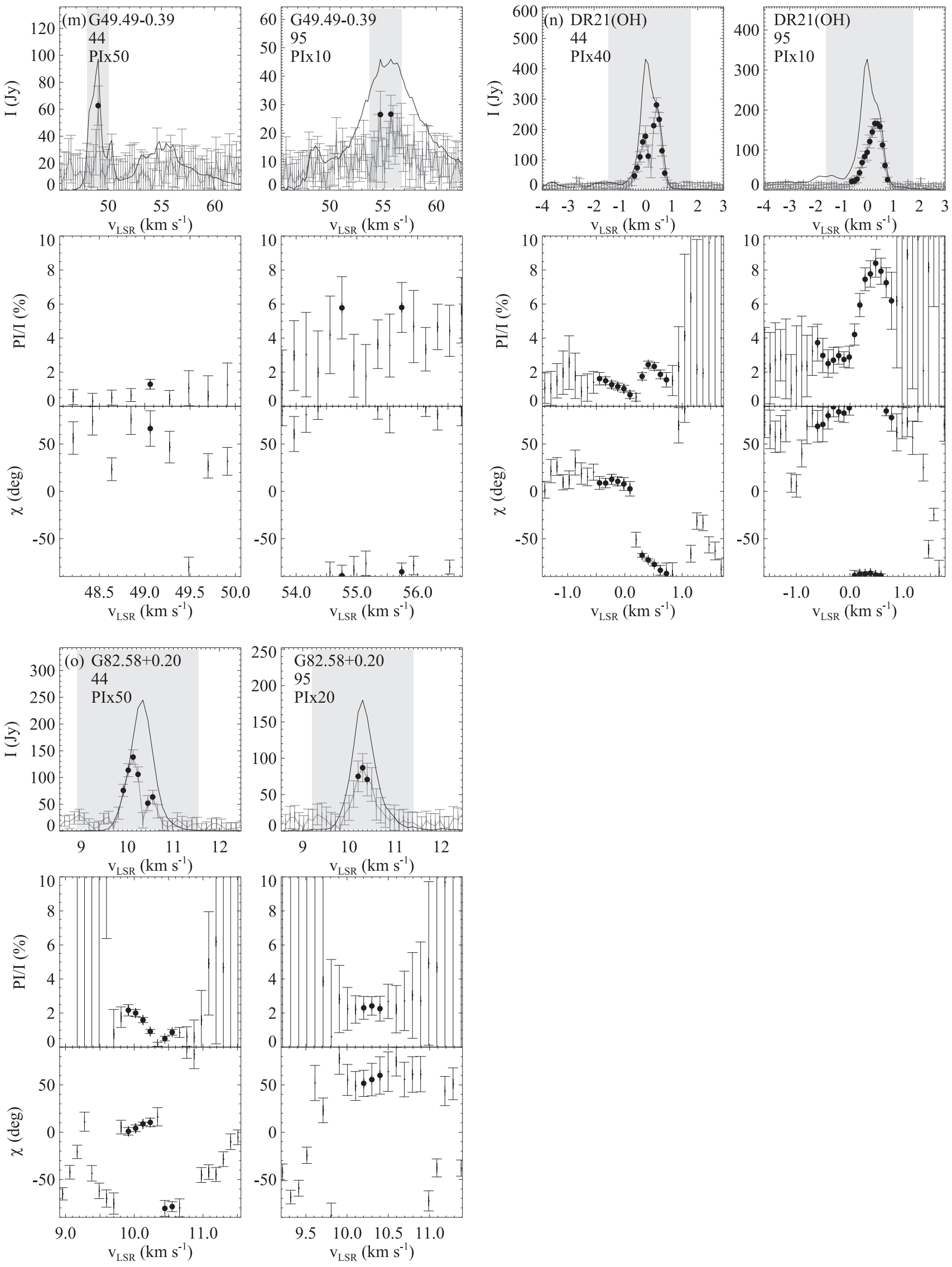

Figure 4. (Continued.) 

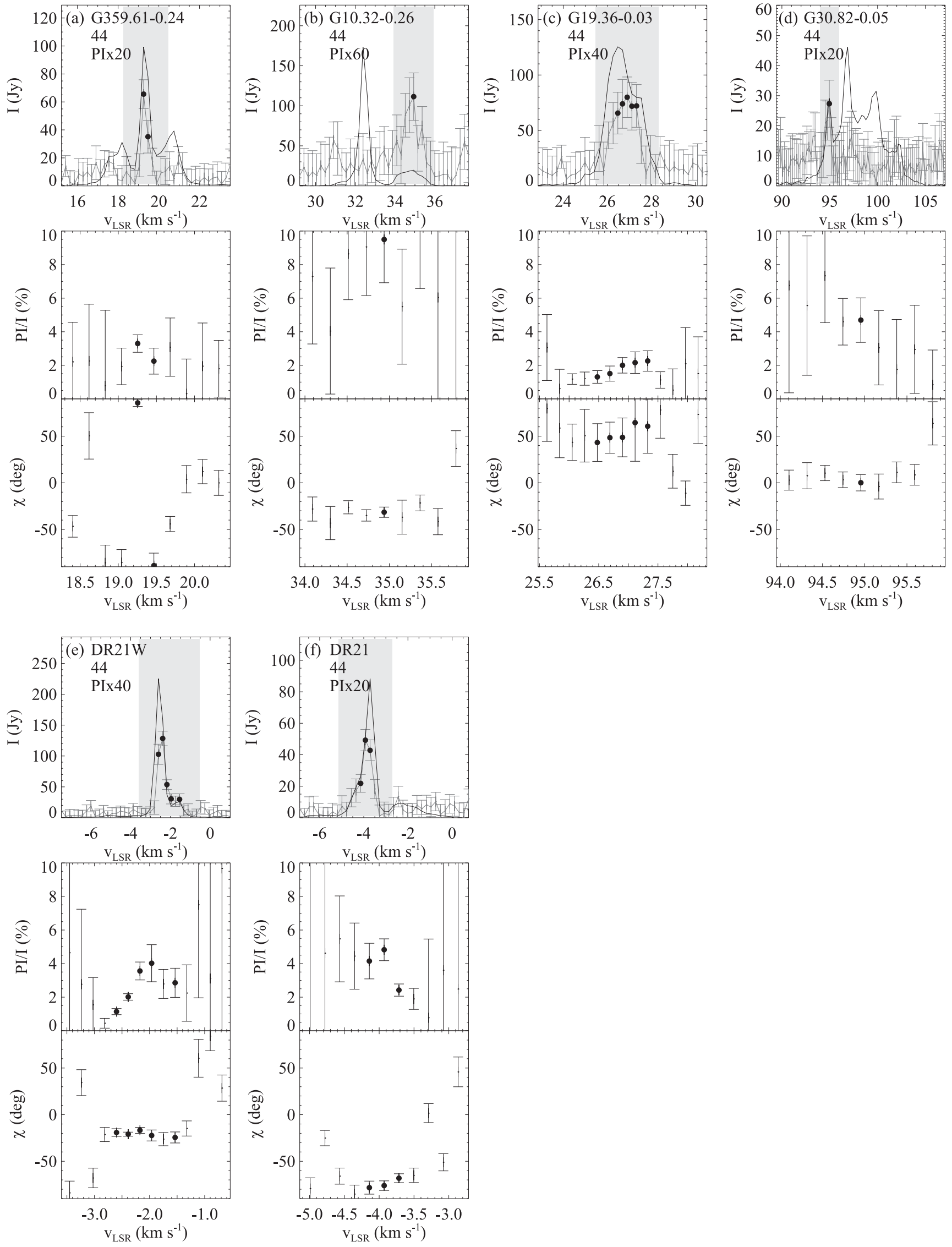

Figure 5. Polarization-detected sources only at $44 \mathrm{GHz}$. 

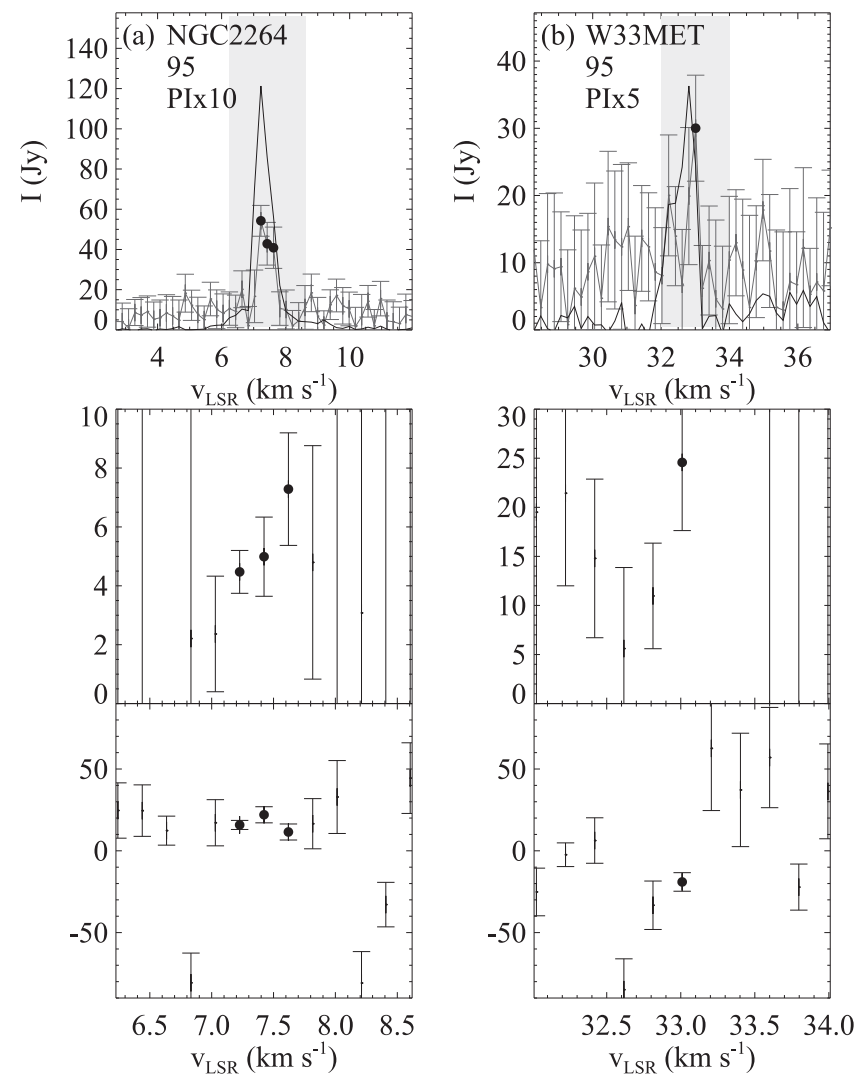

Figure 6. Polarization-detected sources only at $95 \mathrm{GHz}$.

The measured linear polarization fractions in this study are mostly less than $10 \%$. The fractions could be lower limits, because there are several effects reducing the original degree of linear polarization. Linear polarization can be reduced by field line curvature along the propagation direction by destroying the phase coherence of polarization (Elitzur 2002), or by internal Faraday rotation due to the presence of electrons in the magnetized medium along the maser amplification path (Fish \& Reid 2006). However, the effects of internal Faraday rotation are expected to be small (Surcis et al. 2011), so our results are not likely affected by the Faraday rotation. The fraction can also be reduced by depolarization because of combining multiple maser features with different polarization orientations within a large single-dish beam.

None of the five sources detected at $84 \mathrm{GHz}$ or $95 \mathrm{GHz}$ by Wiesemeyer et al. (2004) were detected in our $95 \mathrm{GHz}$ observations, although some targets have high fractional polarizations up to $\sim 9 \%$. This is mainly because of the sensitivity issue. Four (S231, W51Met2, W75S(3), and NGC7538) were weak sources with total fluxes below $20 \mathrm{Jy}$, and could not be detected in our $95 \mathrm{GHz}$ observations with a typical rms of $1.2 \mathrm{Jy}$ and the $3 \sigma$ detection criterion. The other (DR21W) was bright (210 Jy) but was rejected from the detection list because it had a polarization fraction slightly lower than the upper limit $(2 \%)$ of the artificial system polarization at $95 \mathrm{GHz}$.

We have not detected any source with linear polarization above the $30 \%$ that Wiesemeyer et al. (2004) reported at $132 \mathrm{GHz}$ Class I and $157 \mathrm{GHz}$ Class II maser transitions. The highest estimated linear polarization degrees were $40 \%$ and $37 \%$ at 132 and $157 \mathrm{GHz}$, respectively. Linear polarization above $33 \%$ is expected to be rare (Elitzur 2002). It is possible only when the angle between the magnetic field and the ray path is $35^{\circ}<\theta<45^{\circ}$ for isotropic pumping. Nedoluha \& Watson (1990) indicated that, for an angular momentum $J=1-0$ transition, a fractional polarization as high as $100 \%$ is observable when a Zeeman frequency is large, however, about $30 \%$ would be the highest for an angular momentum $J=2-1$ and higher transitions, unless significant anisotropic pumping is present. Wiesemeyer et al. (2004) found that a large fractional linear polarization $\left(P_{L}>33 \%\right)$ is not rare ( 2 out of 10 for Class I and 1 out of 3 for Class II), giving the impression that anisotropic pumping or loss may be commonly achievable, due to an unequal population of the magnetic substates of the maser levels (Nedoluha \& Watson 1990). In the present study, none of the 23 polarization-detected sources show such high linear polarization. We also observed two sources (M8E and L379) that showed the highest fractional polarization $>30 \%$ at $132 \mathrm{GHz}$ in Wiesemeyer et al. (2004). The two had fractional polarizations of $4 \%-10 \%$ at $95 \mathrm{GHz}$. Since transitions at higher frequencies tend to have a higher fraction of polarization, we cannot directly compare our results with those of Wiesemeyer et al. (2004). However, our observational results, which lacked high fractional polarization, out of 23 polarization-detected sources, still suggest that the anisotropic pumping or loss mechanism may not be common for 44 and $95 \mathrm{GHz}$ maser transitions.

\subsection{Comparison of Polarization Properties at 44 and $95 \mathrm{GHz}$}

We have found that the polarization fractions and angles of the 44 and $95 \mathrm{GHz}$ masers are generally well correlated, as shown in Figure 7. Their fractions of linear polarization tend to have a linear correlation and their polarization angles are aligned well. It has been suggested that the two transitions trace similar regions (e.g., Val'tts et al. 2000; Kang et al. 2015). The similar polarization properties of the two lines also confirm that the masers at these two transition lines are indeed experiencing magnetic fields of similar regions.

Our observations show that the degree of linear polarization at $95 \mathrm{GHz}$ is greater than that at $44 \mathrm{GHz}$ for all 15 sources detected at both frequencies, with one exception (G40.25 $-0.19)$. The reduced fractional polarization at the lower frequency transition, compared to the higher frequency transitions, seems to be a general trend in maser polarization observations. For example, McIntosh \& Predmore (1993) and Wiesemeyer et al. (2004) reported the same trend for silicon monoxide and methanol masers, respectively. Several possibilities might explain these observations.

The Faraday rotation has been suggested as an explanation for depolarization at longer wavelengths (Elitzur 2002). The effects of Faraday rotation on the linear polarization of the masers are well described by Fish \& Reid (2006) and Surcis et al. (2011). According to their explanations, internal Faraday rotation due to the free electrons in the path of maser amplification can decrease the linear polarization fraction of the radiation, sometimes completely circularizing it if the Faraday rotation is strong enough (Goldreich et al. 1973). However, Surcis et al. (2011) show that the depolarization due to the internal Faraday rotation for the $6.7 \mathrm{GHz}$ methanol maser is negligible in case of C-shock, and it is not likely significant in the case of J-shock either. The rotation measure changes due to the external Faraday rotation is negligibly small for producing depolarization. When $\mathrm{RM}=1100 \mathrm{rad} \mathrm{m}^{-2}$, the maximum value measured toward pulsars in Han et al. 
Table 3

Linear Polarization Properties

\begin{tabular}{|c|c|c|c|c|c|}
\hline $\begin{array}{l}\text { Source } \\
\text { Name }\end{array}$ & $\begin{array}{c}\text { Transition } \\
(\mathrm{GHz})\end{array}$ & $\begin{array}{l}F \nu \\
(\mathrm{Jy})\end{array}$ & $\begin{array}{c}P_{L} \\
(\%)\end{array}$ & $\begin{array}{l}\chi \\
\left({ }^{\circ}\right)\end{array}$ & $\begin{array}{c}v_{\mathrm{LSR}} \\
\left(\mathrm{km} \mathrm{s}^{-1}\right)\end{array}$ \\
\hline \multirow[t]{2}{*}{ OMC2 } & 44 & $205.6 \pm 2.8$ & $1.3 \pm 0.4$ & $120 \pm 9$ & +11.1 \\
\hline & 95 & $147.8 \pm 5.1$ & $2.0^{\mathrm{a}} \pm 0.7$ & $142 \pm 8$ & +10.9 \\
\hline S255N & 95 & $188.1 \pm 0.7$ & $3.1 \pm 0.3$ & $176 \pm 4$ & +11.0 \\
\hline NGC2264 & 95 & $121.3 \pm 4.0$ & $4.5 \pm 0.9$ & $15 \pm 10$ & +7.2 \\
\hline G357.96-0.16 & 44 & $67.0 \pm 0.4$ & $5.0 \pm 0.6$ & $102 \pm 8$ & -5.0 \\
\hline \multirow{2}{*}{ IRAS18018-2426 } & 44 & $685.0 \pm 5.5$ & $2.3 \pm 0.6$ & $158 \pm 3$ & +10.9 \\
\hline & 95 & $255.3 \pm 1.2$ & $4.0 \pm 0.6$ & $157 \pm 4$ & +10.8 \\
\hline \multirow[t]{2}{*}{ G10.34-0.14 } & 44 & $106.7 \pm 0.7$ & $8.6 \pm 0.5$ & $161 \pm 3$ & +14.6 \\
\hline & 95 & $78.0 \pm 1.8$ & $11.0 \pm 1.0$ & $151 \pm 3$ & +14.6 \\
\hline G10.32-0.26 & 44 & $19.6 \pm 0.4$ & $9.5 \pm 2.6$ & $148 \pm 18$ & +34.9 \\
\hline W33MET & 95 & $24.4 \pm 1.5$ & $24.6 \pm 6.9$ & $160 \pm 19$ & +33.0 \\
\hline G19.36-0.03 & 44 & $99.9 \pm 0.7$ & $2.0 \pm 0.5$ & $48 \pm 21$ & +26.9 \\
\hline \multirow[t]{2}{*}{ L379 } & 44 & $143.8 \pm 1.8$ & $5.7 \pm 0.5$ & $136 \pm 4$ & +17.6 \\
\hline & 95 & $77.6 \pm 3.1$ & $10.1 \pm 1.3$ & $120 \pm 5$ & +17.4 \\
\hline \multirow[t]{2}{*}{ G23.43-0.18 } & 44 & $62.9 \pm 0.8$ & $6.0 \pm 0.5$ & $123 \pm 6$ & +99.6 \\
\hline & 95 & $32.3 \pm 1.1$ & $8.5 \pm 2.7$ & $127 \pm 8$ & +102.3 \\
\hline \multirow[t]{2}{*}{ G27.36-0.16 } & 44 & $129.4 \pm 2.5$ & $1.4 \pm 0.4$ & $98 \pm 13$ & +94.2 \\
\hline & 95 & $74.1 \pm 0.8$ & $7.0 \pm 1.1$ & $113 \pm 5$ & +94.3 \\
\hline \multirow[t]{2}{*}{ G29.91-0.03 } & 44 & $414.9 \pm 2.7$ & $1.1 \pm 0.5$ & $171 \pm 6$ & +98.2 \\
\hline & 95 & $220.9 \pm 4.0$ & $3.4 \pm 0.5$ & $161 \pm 4$ & +98.2 \\
\hline G30.82-0.05 & 44 & $29.1 \pm 0.6$ & $4.7 \pm 1.3$ & $0 \pm 18$ & +95.0 \\
\hline \multirow[t]{2}{*}{ G40.25-0.19 } & 44 & $52.0 \pm 0.6$ & $5.7 \pm 0.6$ & $110 \pm 8$ & +74.0 \\
\hline & 95 & $116.0 \pm 3.5$ & $4.6 \pm 0.5$ & $96 \pm 4$ & +72.7 \\
\hline G49.49-0.39 & 44 & $97.1 \pm 2.0$ & $1.3 \pm 0.3$ & $66 \pm 19$ & +49.1 \\
\hline
\end{tabular}

Note. The presented values of $F_{\nu}, P_{L}, \chi$, and $v_{\mathrm{LSR}}$ are all determined in the channel with the peak polarized intensity.

${ }^{a}$ We note that the $95 \mathrm{GHz}$ polarization fractions of OMC2 $(2.0 \%)$ and $\mathrm{G} 82.58+0.20(2.5 \%)$ are the same or close to the upper limit $(2 \%)$ of the artificial polarization of the system at $95 \mathrm{GHz}$. Thus, we cannot discard the possibility that their detections were affected by the artifacts of the system.

(2006), is adopted, the angle due to the Faraday rotation is only $3^{\circ}$ at $44 \mathrm{GHz}$, which is in general smaller or comparable to the angle measurement error. Thus, the Faraday rotation cannot explain the lower polarization fraction at $44 \mathrm{GHz}$.

Another possibility is the lower $J$ transition data averaging over a larger volume of the medium due to the larger beam than the other transitions (McIntosh \& Predmore 1993). Variation of physical conditions in a larger volume may reduce the fractional polarization. However, the similarities in total flux and polarization properties in our observations suggest that both transitions are likely covering similar maser components. In addition, according to the previous VLA observations (Kogan \& Slysh 1998; Kurtz et al. 2004) of methanol masers, a case of the $44 \mathrm{GHz}$ masers being distributed over an area wider than the KVN beam, $30^{\prime \prime}$ at $95 \mathrm{GHz}$, is rare. Thus, different a volume does not seem to be the main cause for the difference in fractional polarization.

The fractional linear polarization of the 44 and $95 \mathrm{GHz}$ transitions could be intrinsically different. Pérez-Sánchez \&
Vlemmings (2013) calculated the fractional linear polarization as a function of the emerging brightness temperature for some rotational transitions of $\mathrm{SiO}, \mathrm{H}_{2} \mathrm{O}$, and $\mathrm{HCN}$ masers. In their calculation, the higher $J$ transitions have somewhat lower polarization percentages, which is mainly due to the averaging over more magnetic substates. However, this assumes similar levels of stimulated emission rate, $R$. As also shown in Nedoluha \& Watson (1990), the linear polarization fraction is a function of the degree of maser saturation, $R / \Gamma$, a ratio of stimulated emission rate to decay rate, and the angle $\theta$ between the line of sight (LOS) and the magnetic field orientation. One of their main findings is that the polarization characteristics for high $J$ transitions are similar in quality and quantity, suggesting that the behaviors of the $7_{0}-6_{1} A+$ and $8_{0}-7_{1} A+$ transitions would be similar as long as they have similar saturation levels and $\theta$. The angle $\theta$ is likely to be similar for both transitions. Although not much is known about the decay rate of the masers (Vlemmings et al. 2010), in our case, $R$ is also similar for both maser transitions. The stimulated emission rate $R$ is 

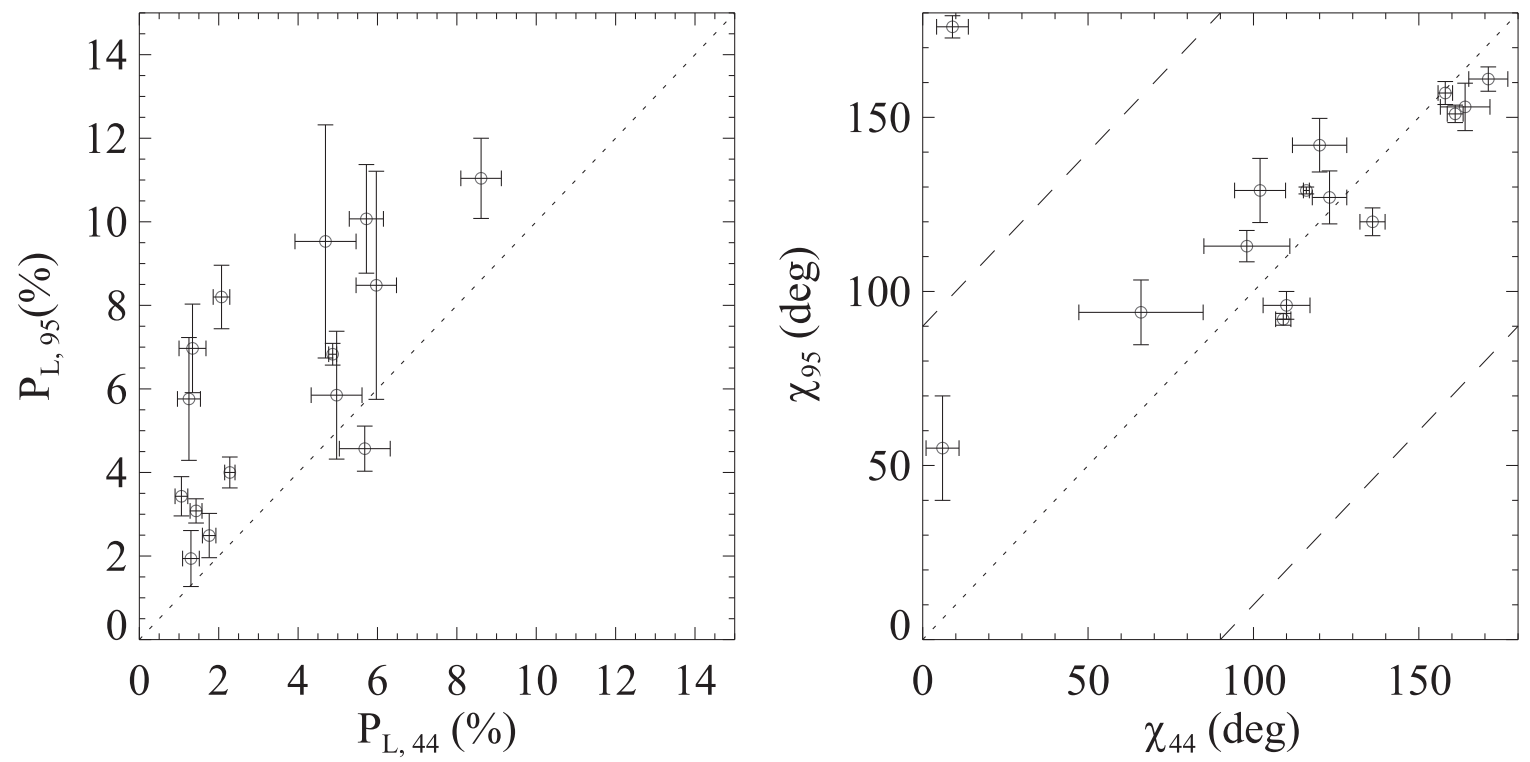

Figure 7. $44 \mathrm{GHz}$ vs. $95 \mathrm{GHz}$ masers in polarization fraction, $P_{L}$ (left), and angle, $\chi$ (right), for the sources detected in both transitions at the same velocities. Errors of $1 \sigma$ are presented. The dotted lines show the cases when the polarization degree and angles of the two transitions are the same. The dashed lines in the right panel show the cases when the angels are $90^{\circ}$ different.

given by $R \sim \frac{A k T_{b} \Delta \Omega}{4 \pi h \nu}$, where $\mathrm{A}$ is the Einstein coefficient of the involved transition, $k$ and $h$ are the Boltzmann and Planck constants, $\nu$ is the maser frequency, $T_{\mathrm{b}}$ is the brightness temperature, and $\Delta \Omega$ is the relation between the real angular size of the masing cloud and the observed angular size (PérezSánchez \& Vlemmings 2013). The Einstein coefficients are $A_{44 \mathrm{GHz}} \sim 2.74 \times 10^{-7} \mathrm{~s}^{-1}$ and $A_{95 \mathrm{GHz}} \sim 2.87 \times 10^{-6} \mathrm{~s}^{-1}$ (B. Lankhaar et al., 2016, in preparation). The brightness temperature $T_{\mathrm{b}}$ can be estimated using $T_{\mathrm{b}}=S_{\nu} \Sigma^{-2} \zeta_{\nu}$, where $S_{\nu}$ is the observed flux density, $\Sigma$ is the maser angular size, and $\zeta_{\nu}$ is a constant factor that includes a proportionality factor obtained for a Gaussian shape. This factor $\zeta_{\nu} \propto \nu^{-2}$, and is $\sim 3.16 \times 10^{8}$ at $44 \mathrm{GHz}$ and $\sim 6.77 \times 10^{7}$ at $95 \mathrm{GHz}$, respectively (Pérez-Sánchez \& Vlemmings 2013). Thus, $T_{\mathrm{b}}$ is roughly five times larger at $44 \mathrm{GHz}$ than at $95 \mathrm{GHz}$ when assuming the same physical size and flux. When additionally the same beaming angle is assumed, the stimulated emission rate $R_{44 \mathrm{GHz}} \approx 0.96 R_{95 \mathrm{GHz}}$. As the flux of the $95 \mathrm{GHz}$ masers is found to be $\sim 50 \%-80 \%$ of that of the $44 \mathrm{GHz}$ masers, $R_{44 \mathrm{GHz}}$ for the individual masers is unlikely to be more than $\sim 2 \times R_{95 \mathrm{GHz}}$. Saturation effects are thus unlikely to contribute to the observed differences unless the physical sizes of the masers and/or the beaming angles of the two transitions are very different. Related to this, the dependence of the linear polarization on the ratio between the Zeeman splitting rate and the stimulated emission rate, $g \Omega / R$, can still contribute to the observed differences between the two maser transitions (Nedoluha \& Watson 1990), provided $g \Omega$ is sufficiently different between the two transitions. A full understanding of the intrinsic cause of a higher polarization fraction in the higher frequency transition line will require further support from future theoretical studies.

\subsection{Association with Outflows or Galactic-scale Magnetic Fields}

Class I methanol masers are collisionally excited and thought to occur in the areas where the methanol molecular abundance is enhanced by the outflow shock heating of grain mantles (Menten 1991). If they arise in the regions compressed by outflow shock, then the observed polarization angles could be associated with the orientations of outflows. We have searched for the references related to the outflows of the 23 polarization-detected sources using the SIMBAD website (Wenger et al. 2000). Many sources have references indicating the existence of outflows, such as $\mathrm{H}_{2}$ maps, extended green objects, or outflow tracing molecular line observations, but the directions of outflows are not obvious in many cases. Among them, we have found seven sources, i.e., OMC2, S255N, NGC2264, G49.49-0.39 (W51 e2), DR21W, DR21, and DR21 $(\mathrm{OH})$, where the direction of a maser-associated outflow is rather simple. IRAS18018-2426, GGD27, L379, and G30.82-0.05 (W42 main) have been mapped in CO transition lines as well, but the connection between the maser and outflow is not obvious either, due to the outflow direction being in the LOS or due to the complicated multiple-outflow morphology (Kelly \& MacDonald 1996; Zhang et al. 2005; FernándezLópez et al. 2013; Sridharan et al. 2014).

We have compared the polarization angles at $44 \mathrm{GHz}$ for 6 sources and at $95 \mathrm{GHz}$ for NGC2264, which is not detected at $44 \mathrm{GHz}$, with the orientations of outflows available in the literature. The position angles (PAs) of their outflows, their references, and the angles between the polarization angle and the outflow are summarized in Table 4. Regarding the errors of $\left|\chi-\mathrm{PA}_{\text {out }}\right|$, we took the measurement errors of the polarization angles, but the errors would be larger considering that the errors of outflows are normally larger. For example, the conventional error of outflow is considered to be $15^{\circ}$ in Surcis et al. (2013). There are some sources with polarization angles that are almost perpendicular to the outflow direction, e.g., OMC2 and DR21W. However, in general the association between outflows and polarization angles is not apparent.

Although the sample size is small, we tried the KolmogorovSmirnov (K-S) statistics to see whether the angle differences are similar to or different from the projected angles of aligned, perpendicular, or randomly aligned samples, by comparing the observed angle difference to the results from the Monte Carlo simulations as discussed in Hull et al. (2014). The simulation 
Table 4

Outflow Orientations and Polarization Angles

\begin{tabular}{lrccc}
\hline \hline Source Name & $\chi$ & $\mathrm{PA}_{\text {out }}$ & $\mid \chi-\mathrm{PA}_{\text {out }}$ & Reference \\
\hline OMC2 & $120^{\circ}$ & $30^{\circ}$ & $90^{\circ} \pm 9^{\circ}$ & Williams et al. (2003) \\
S255N & $9^{\circ}$ & $51^{\circ}$ & $42^{\circ} \pm 5^{\circ}$ & Wang et al. (2011) \\
NGC2264 & $15^{\circ}$ & $175^{\circ}$ & $20^{\circ} \pm 10^{\circ}$ & Schreyer et al. (2003) \\
G49.49-0.39 (W51 e2) & $66^{\circ}$ & $137^{\circ}$ & $71^{\circ} \pm 19^{\circ}$ & Shi et al. (2010) \\
DR21W & $159^{\circ}$ & $70^{\circ}$ & $9^{\circ} \pm 4^{\circ}$ & Garden et al. (1991) \\
DR21(OH) & $109^{\circ} / 36^{\circ}$ & $113^{\circ}$ & $4^{\circ} \pm 3^{\circ} / 77^{\circ} \pm 3^{\circ}$ & Zhang et al. (2014) \\
DR21 & $103^{\circ}$ & $150^{\circ}$ & $47^{\circ} \pm 8^{\circ}$ & Garden et al. (1991) \\
\hline
\end{tabular}

Note. $\chi$ at $44 \mathrm{GHz}$ is used for comparison, except for NGC2264, which is polarization-detected only at $95 \mathrm{GHz}$. Position angles of outflows (PA $\mathrm{out}_{\text {) }}$ are estimated counterclockwise from the north, the same way that the polarization angles of $44 \mathrm{GHz}$ masers are measured. The reference for the outflow orientation is noted in the Reference column. The error of the PA difference in column 4 is adopted from the $44 \mathrm{GHz}$ maser polarization angle measurement error. DR21(OH) has two perpendicular polarization angles, resulting in two perpendicular $\left|\chi-\mathrm{PA}_{\text {out }}\right|$ values.
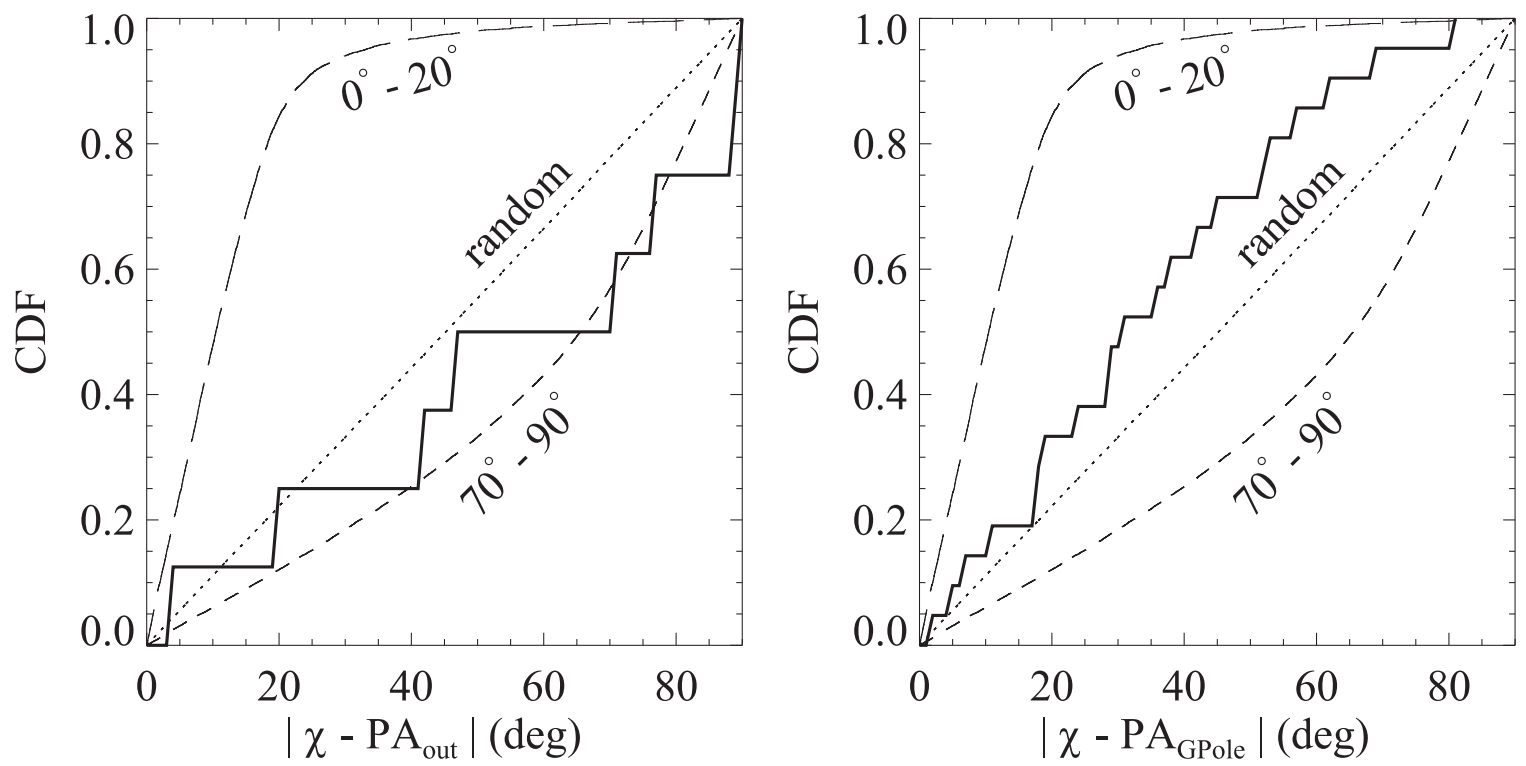

Figure 8. The thick solid lines show the cumulative distribution functions (CDFs) of the angles between the polarization angles and the orientations of outflows (left) and those of the Galactic pole (right). The dotted line and dashed and long-dashed curves are the CDFs of projected angles from Monte Carlo simulations where two vectors are randomly distributed, perpendicular to, and well-aligned with each other, respectively.

that randomly selects pairs of vectors in three dimensions gives the same distribution when it is projected on the plane of the sky. However, the projection effects increase the scatter of two dimensional (2D) projected angles in other cases. For the aligned case, we select pairs of vectors that are aligned within $20^{\circ}$ of one another among the random pairs in three dimensions and calculate their angles projected onto the plane of the sky. For the perpendicular case, we selected pairs of vectors separated by $70^{\circ}-90^{\circ}$, and projected them. The projection effects are more important in the perpendicular case than those in the aligned case. The cumulative distribution functions (CDFs) of the 2D projected angles from simulations are compared with the CDFs of the observed angle difference between the outflow direction and the polarization angle in the left panel of Figure 8.

The probabilities of the observed $\left|\chi-\mathrm{PA}_{\text {out }}\right|$ and the simulated projected angles being different or similar that are derived from the $\mathrm{K}-\mathrm{S}$ tests are given in Table 5 . The $\mathrm{K}-\mathrm{S}$ tests rule out the scenario where the outflows and polarization angles are tightly aligned $(P<0.01)$. The probability of them being perpendicular $(P=0.7)$ appears to be higher than that of them being random $(P=0.4)$. Magnetic fields could be either parallel or perpendicular to the polarization angle depending on
Table 5

Probability of the K-S Test

\begin{tabular}{ccc}
\hline \hline & $\left|\chi-\mathrm{PA}_{\text {out }}\right|$ & $\left|\chi-\mathrm{PA}_{\mathrm{GPole}}\right|$ \\
\hline random & $P=0.4$ & $P=0.3$ \\
$0^{\circ}-20^{\circ}$ & $<0.01$ & $<0.01$ \\
$70^{\circ}-90^{\circ}$ & 0.7 & $<0.01$ \\
\hline
\end{tabular}

Note. Probability is a value between 0 and 1 giving the significance level of the $\mathrm{K}-\mathrm{S}$ statistics. Small values of probability indicate that the CDFs of the two data sets are significantly different from one another.

the angle between the magnetic field and the ray path, $\theta$. If we simply assume that methanol masers are strongly saturated $\left(R / \Gamma>10^{2}\right)$ because they are strong, then the polarization angle is perpendicular to the magnetic field regardless of $\theta$ (Nedoluha \& Watson 1990). If we perform the K-S tests for $\left|B-\mathrm{PA}_{\text {out }}\right|$ and take into account the above assumption, the $\mathrm{K}-\mathrm{S}$ tests returns $P=0.5$ for random, and $P<0.1$ for aligned and perpendicular cases of simulations. Considering the ambiguity of the magnetic field direction derived from the polarization angle, the limited number of samples, and the difficulty of distinguishing the projected angle distribution 

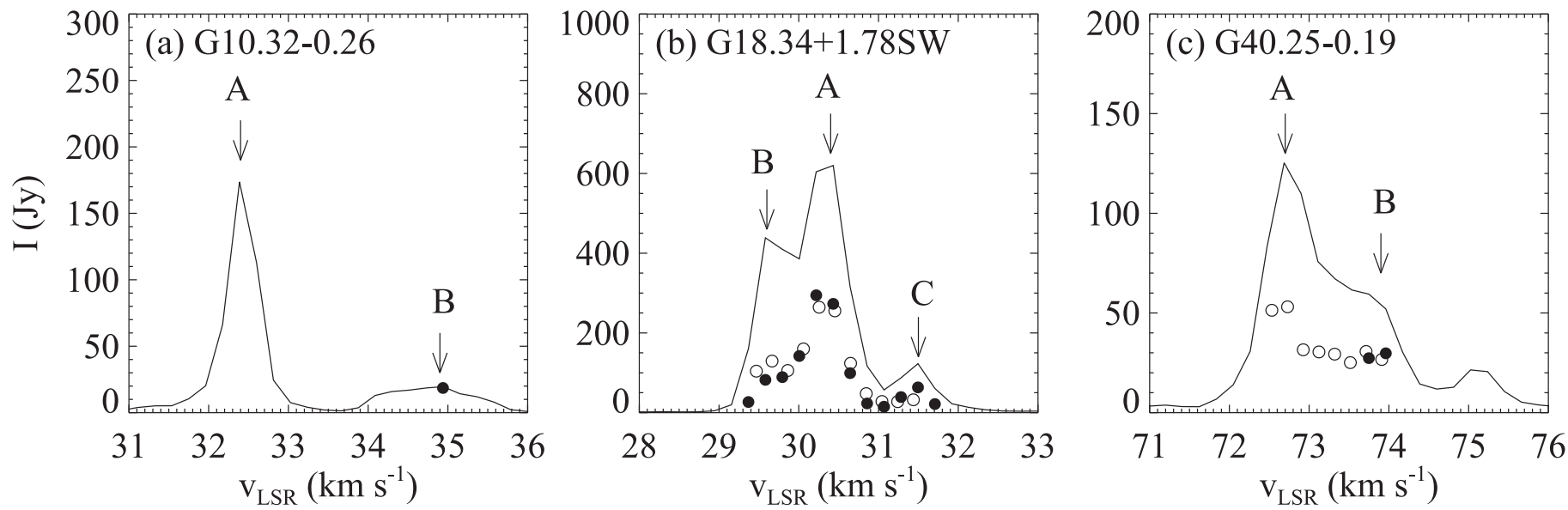

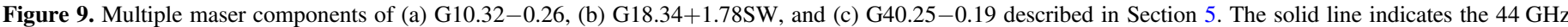

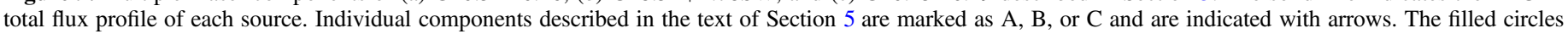
indicate the points with $\mathrm{PI}_{44 \mathrm{GHz}}>3 \sigma$ after being multiplied times 10 The open circles are the same but at $95 \mathrm{GHz}$.

from the random and $70^{\circ}-90^{\circ}$ simulations, we cannot conclude whether magnetic fields/polarization angles are randomly aligned or misaligned with outflows; however, we can at least rule out the scenario in which they are tightly aligned.

Maser-emitting regions are very small in size, so the magnetic fields traced by masers are not expected to be associated with the magnetic field on Galactic or molecular cloud scales. However, there have been some studies showing that the field orientations measured from the Zeeman effect of $\mathrm{OH}$ masers in the star-forming regions have a positive correlation with the Galactic-scale magnetic field measured from Faraday rotations (e.g., Fish \& Reid 2006; Han \& Zhang 2007; Noutsos 2012). Since Class I methanol masers occur in a medium with a similar or slightly smaller density, and at a larger distance from the central stars than the $\mathrm{OH}$ masers, we have investigated the possibility of an association between the linear polarization of the $44 \mathrm{GHz}$ methanol masers and the orientation of the Galactic pole, which could be related to the large-scale magnetic field.

We apply the same analysis that is done for the outflows. As seen in the right panel of Figure 8 , the CDF of the angle between the polarization angle and the direction of the Galactic pole, $\left|\chi-\mathrm{PA}_{\mathrm{GPole}}\right|$, is neither similar to the $\mathrm{CDF}$ of $0^{\circ}-20^{\circ}$ simulation nor to that of the $70^{\circ}-90^{\circ}$ simulation. The $\mathrm{K}-\mathrm{S}$ tests also retune $P<0.01$ for these two cases (Table 5), ruling out the scenario of the polarization angles having preference on the directions of the Galactic plane or pole.

\section{DISCUSSION ON SOURCES WITH PECULIAR POLARIZATION PROPERTIES}

We discuss some interesting targets with unique polarization characteristics in detail.

\subsection{G10.32-0.26}

The linear polarization of G10.32-0.26 is detected only at $44 \mathrm{GHz}$ (see Figure 5(b)). It is peculiar because the linear polarization is not detected in the brightest peak of the total flux profile at $v_{\mathrm{LSR}} \sim 32.5 \mathrm{~km} \mathrm{~s}^{-1}$ (A component in Figure 9(a)) with an upper limit of $0.9 \%$, but its secondary peak at $v_{\text {LSR }}$ $\sim 35 \mathrm{~km} \mathrm{~s}^{-1}$ (B component in Figure 9(a)) shows polarization. We confirmed this trend by observing them in two or more epochs. As mentioned in Section 2.1, our observations were
Table 6

Polarization Properties of G18.34+1.78SW

\begin{tabular}{lcccrc}
\hline \hline $\begin{array}{l}v_{\mathrm{LSR}} \\
\left(\mathrm{km} \mathrm{s}^{-1}\right)\end{array}$ & $\begin{array}{c}\text { Transition } \\
(\mathrm{GHz})\end{array}$ & $\begin{array}{c}I \\
(\mathrm{Jy})\end{array}$ & \multicolumn{1}{c}{$\begin{array}{c}P_{L} \\
(\%)\end{array}$} & \multicolumn{1}{c}{$\begin{array}{c}\chi \\
\left({ }^{\circ}\right)\end{array}$} & $\begin{array}{c}\Delta v_{\mathrm{FWHM}} \\
\left(\mathrm{km} \mathrm{s}^{-1}\right)\end{array}$ \\
\hline+30.3 & 44 & 678. & $4.8 \pm 0.1$ & $118 \pm 1$ & 0.5 \\
& 95 & 411. & $6.8 \pm 0.2$ & $130 \pm 1$ & 0.6 \\
+29.6 & 44 & 471. & $2.0 \pm 0.1$ & $96 \pm 1$ & 0.4 \\
& 95 & 346. & $3.6 \pm 0.2$ & $139 \pm 1$ & 0.5 \\
+31.4 & 44 & 124. & $5.4 \pm 0.4$ & $117 \pm 2$ & 0.4 \\
& 95 & 50. & $6.2 \pm 2.4$ & $132 \pm 8$ & 0.5 \\
\hline
\end{tabular}

Note. Spectra with $0.1 \mathrm{~km} \mathrm{~s}^{-1}$ resolution were used for the Gaussian fit. The $v_{\text {FWHM }}$ presented is the fitting result of a polarized intensity profile, which is $0.2 \mathrm{~km} \mathrm{~s}^{-1}$ smaller in maximum than the total intensity profile.

targeted for the brightest features in the total intensity. If the weaker feature is largely displaced from the brightest one, for example, by $30^{\prime \prime}$, that could produce artificial polarization of up to $2 \%$. The displacement of the $35 \mathrm{~km} \mathrm{~s}^{-1}$ component from the brightest peak is less than $2^{\prime \prime}$, according to the VLBI observation using the KVN and VERA combined array (KaVA; M. K. Kim et al. 2016, in preparation) and its observed degree of polarization is $4 \%-9 \%$, well above $2 \%$. Thus, these detections are very likely real.

\section{2. $G 18.34+1.78 S W$}

$\mathrm{G} 18.34+1.78 \mathrm{SW}$ is one of the brightest methanol masers known in the Galaxy and first detected in the KVN single-dish maser surveys (K. T. Kim et al. 2016, in preparation). It is associated with millimeter-core MM2 in a massive star-forming region, IRAS 18151-1208 (Marseille et al. 2008). It is imaged by the $\mathrm{KaVA}$ at an angular resolution of $\sim 2$ mas (Matsumoto et al. 2014).

The single-dish total intensity spectra of G18.34+1.78SW show three maser features with peak velocities of +30.3 (A) +29.6 (B), and $+31.4 \mathrm{~km} \mathrm{~s}^{-1}$ (C in Figure 9(b)) in both transitions, all of which show linearly polarized emission in both transitions (see also Figure 4(f)). The velocities of these three components agree well with the findings of Matsumoto et al. (2014), although much of the total flux was missed in the VLBI image. The polarization properties of these three components are summarized in Table 6 . We used a function 
with three Gaussian components to fit the $0.1 \mathrm{~km} \mathrm{~s}^{-1}$ resolution spectra. The line widths measured in the polarization profile range between 0.4 and $0.6 \mathrm{~km} \mathrm{~s}^{-1}$, while it is $0.2 \mathrm{~km} \mathrm{~s}^{-1}$ wider when measured in the total flux profile.

This object has $P_{L}=2.0 \%-6.8 \%$ in two transitions, and all three components show slightly higher polarization at $95 \mathrm{GHz}$, which is generally observed for other sources. The polarization angles of the brightest peak are similar at 44 and $95 \mathrm{GHz}$, i.e., $12^{\circ}$ different, but the difference is beyond the measured error. The angle difference ranges up to $43^{\circ}$ in the second brightest peak. The fact that the polarization angle differences are not consistent for the three maser features at nearly the same position, at most 100 mas apart (Matsumoto et al. 2014), indicates that neither rotation measure nor the beam size can explain the difference in the polarization properties of the 44 and $95 \mathrm{GHz}$ transitions. It may be a combination of the magnetic field morphology and the saturation level difference between the two transition lines, as discussed in Section 4.2. High-resolution line polarimetry using the VLA or ALMA will help us to understand the underlying physics.

\section{3. $G 40.25-0.19$}

The single-dish total intensity profiles of G40.25-0.19 show multiple maser components at $v_{\mathrm{LSR}}=+72-+75.5 \mathrm{~km} \mathrm{~s}^{-1}$ at both transitions (see Figures 4(1) and 9(c)). This target is interesting because it shows linear polarization degrees of $4.5 \%-7 \%$ at $95 \mathrm{GHz}$ in all maser components at $v_{\mathrm{LSR}}$ $=+72-+75.5 \mathrm{~km} \mathrm{~s}^{-1}$, but only a secondary bright feature at $v_{\mathrm{LSR}} \sim 73.8 \mathrm{~km} \mathrm{~s}^{-1}$ (B in Figure 9(c)) is detected at $44 \mathrm{GHz}$. The upper limit of the brightest peak at $v_{\text {LSR }} \sim 72.5 \mathrm{~km} \mathrm{~s}^{-1}$ (A) is $0.8 \%$. The difference in polarization properties could also be related to the maser saturation level for this source.

\section{4. $\mathrm{DR} 21(\mathrm{OH})$}

The $44 \mathrm{GHz}$ masers of DR21(OH) have been imaged with the VLA (Kogan \& Slysh 1998; Kurtz et al. 2004). The VLA results show that there are two groups of masers, one at $v_{\mathrm{LSR}}$ $\sim 0 \mathrm{~km} \mathrm{~s}^{-1}$ and the other at $v_{\mathrm{LSR}} \sim-4 \mathrm{~km} \mathrm{~s}^{-1}$, about $20^{\prime \prime}$ apart from each other. These two groups of masers are located at the end of approaching and receding outflow in the DR21(OH) region (Zhang et al. 2014), supporting the theory that Class I methanol masers are stimulated by outflows. Our observation was pointed to the brightest peak at $v_{\mathrm{LSR}} \sim 0 \mathrm{~km} \mathrm{~s}^{-1}$ that contains $\sim 80 \%$ of the total flux and is located at the end of an receding outflow lobe. This feature has an elongated, arc-shape morphology in the VLA map.

The linear polarization of DR21(OH) shows features indicating depolarization and a sudden flip of polarization angle in the $44 \mathrm{GHz}$ transition line. The total intensity and the polarized emission of DR2 $1(\mathrm{OH})$ at $44 \mathrm{GHz}$ show two maser components, one at $v_{\mathrm{LSR}}=-0.5-+0.2 \mathrm{~km} \mathrm{~s}^{-1}$ and the other at $v_{\mathrm{LSR}}=+0.2-+0.8 \mathrm{~km} \mathrm{~s}^{-1}$, which are indicated as $\mathrm{A}$ and $\mathrm{B}$ regions in panel (a) of Figure 10. Their polarization fractions are similar, $\sim 2 \%$, while their polarization angles differ by $\sim 90^{\circ}$. At the velocity where the two components overlapped, the fraction of linear polarization drops to minimum.

Taking into account the previous observations, elongated masers aligned along a curved receding shock front with the magnetic field compressed along the shock can explain the observed profiles. Such a flip of polarization angle is expected around $\theta=55^{\circ}$ in maser theories (e.g., Goldreich et al. 1973), and has been observed previously in $\mathrm{SiO}$ masers by Kemball \& Diamond (1997) and in $\mathrm{H}_{2} \mathrm{O}$ by Vlemmings \& Diamond (2006). In these observations, the angle flip appeared in a single maser feature, which was interpreted as evidence of a curved magnetic field, with $\theta$ being close to the van Vleck angle, i.e., $\theta=55^{\circ}$. In our case, the flip happens in two different maser features separated in velocity by a single-dish spectrum, but the underlying physics could be the same. If the two maser features are located in a curved magnetic field aligned along the shock front receding from us, each of their $\theta$ being $\theta<55^{\circ}$ and $\theta>55^{\circ}$, then, they would produce a $90^{\circ}$ angle flip as well as a LOS velocity shift. One implication of this explanation is that the $44 \mathrm{GHz}$ masers of DR21(OH) are unsaturated, or only moderately saturated according to the simulation of Nedoluha \& Watson (1990). In the upper panels of Figure 3 in their paper, the polarization angle ( $\phi$ in their paper) flips by the change of $\theta$ only when $\log R / \Gamma \lesssim 1$. When the maser is highly saturated, e.g., when $\log R / \Gamma=3$, the polarization angle is not seriously affected by $\theta$ but rather constant.

The polarization angle can also differ by as much as $90^{\circ}$ when the saturation levels of the two maser components are different. As seen in Figure 3 of the above paper, for example, if the saturation levels of the two components are $\log R / \Gamma=1$ and 3 , respectively, the two components will show $90^{\circ}$ of polarization angle difference even for the same field orientation with $\theta=15^{\circ}$. How much the saturation levels of the same $44 \mathrm{GHz}$ transition line can differ is another issue. Assuming that the rare factor $\Gamma$ is similar, $R$ could be determined by the physical size and flux of maser components. The observed fluxes differ by less than a factor of two, while their physical sizes cannot be determined with the current observations.

The angle flip is not observed in the $95 \mathrm{GHz}$ transition line profile (see Figure $4(n)$ ). While the polarization angle of the $-0.5-+0.1 \mathrm{~km} \mathrm{~s}^{-1}$ component at $95 \mathrm{GHz}$ is almost perpendicular to that of the $44 \mathrm{GHz}$ maser, the angle of the $+0.3-+0.7 \mathrm{~km} \mathrm{~s}^{-1}$ component is similar to that of the $44 \mathrm{GHz}$ maser. If the angle flip in the $44 \mathrm{GHz}$ maser is due to the van Vleck angle crossing, the polarization angles of the $95 \mathrm{GHz}$ maser being relatively constant may imply that the $95 \mathrm{GHz}$ transition is more highly saturated than the $44 \mathrm{GHz}$ transition, a condition in which the PA is always perpendicular to the magnetic field regardless of $\theta$, as demonstrated by Nedoluha \& Watson (1990). The saturation levels of masers are difficult to measure. Future high-resolution observations and theoretical studies dedicated to methanol maser lines will be able to reveal the morphology, field orientation, and kinematics of the DR21 $(\mathrm{OH})$ region as well as the involved maser physics.

\section{5. $G 82.58+0.20$}

The polarization profiles of $\mathrm{G} 82.58+0.20$ present polarization intensity and angle variations similar to those of DR21 $(\mathrm{OH})$ at both transition lines. They show polarization angle flip and a reduced polarization fraction at the velocities where the two velocity components overlapped in the $44 \mathrm{GHz}$ transition line, as indicated in the $\mathrm{A}$ and $\mathrm{B}$ regions in panel (b) of Figure 10. This may imply the existence of elongated masers associated with a curved magnetic field orientation in a compressed medium. This target would be a good candidate for high-resolution polarimetry observations. 

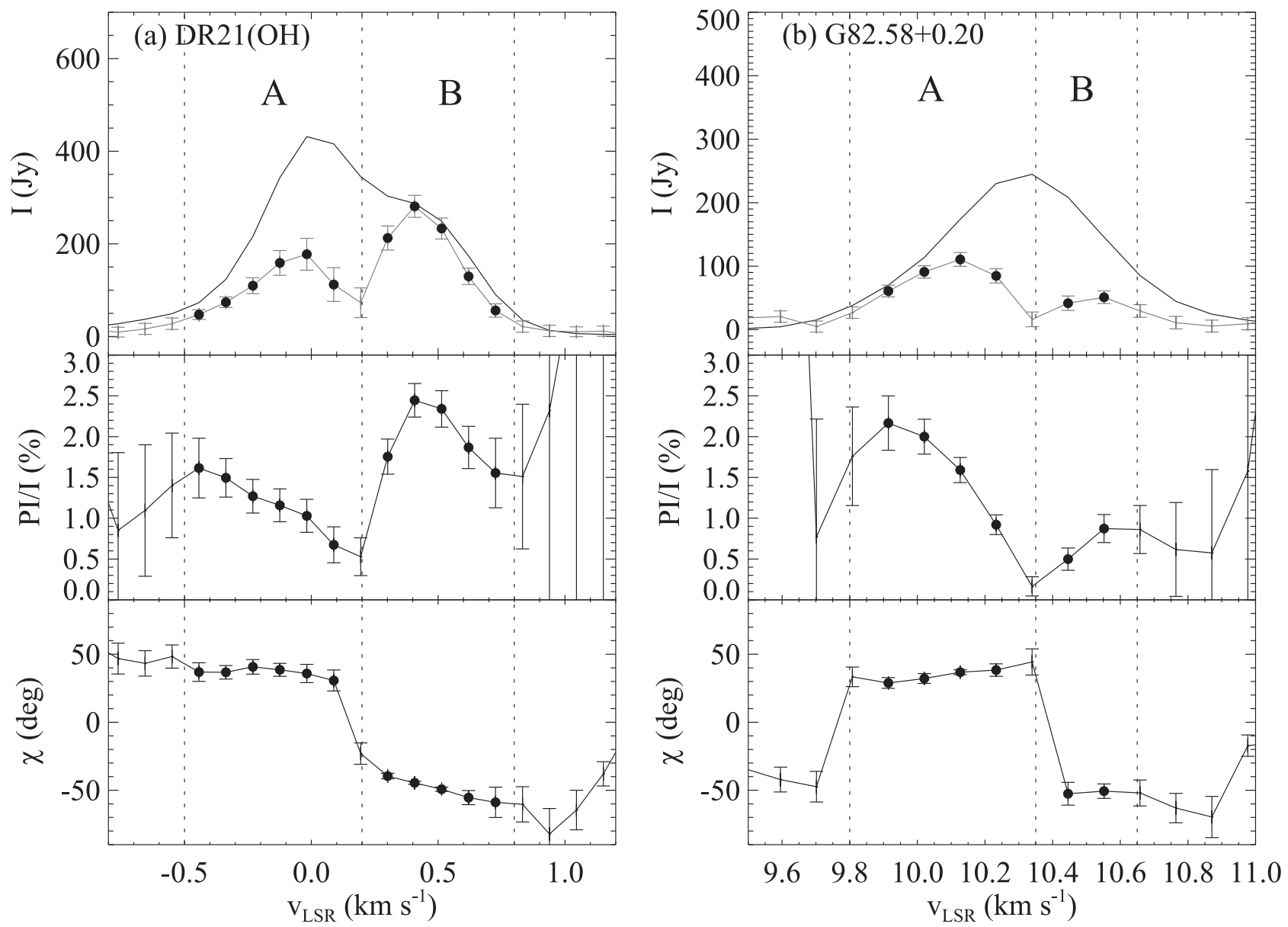

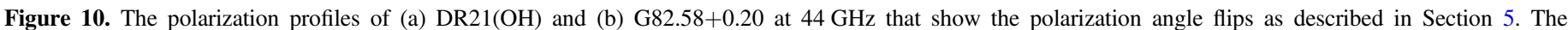

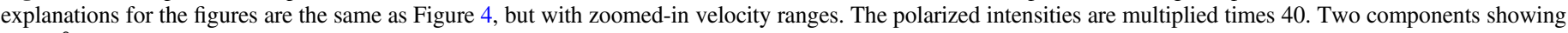
the $90^{\circ}$ angle flips are marked as A and B after being divided by the dotted lines.

\section{SUMMARY}

We performed the first simultaneous, comprehensive study of the linear polarization of a large sample of 39 bright Class I methanol maser sources in the 44 and $95 \mathrm{GHz}$ transitions. The main findings of this study are as follows.

1. We detected 23 sources (59\%) in at least 1 transition and 15 sources $(38 \%)$ in both transitions. Their errorweighted mean degrees of polarizations are $2.7 \pm 0.3 \%$ and $4.8 \pm 0.1 \%$ at 44 and $95 \mathrm{GHz}$, respectively. The linear polarization of the $44 \mathrm{GHz}$ methanol maser transition was first detected in this study.

2. We compared the polarization properties of the 44 and $95 \mathrm{GHz}$ transition lines. We found that their polarization fractions are linearly correlated, but the emission from the $95 \mathrm{GHz}$ tends to be more linearly polarized than that from the $44 \mathrm{GHz}$ transition line. We suggest that this is not likely due to an external reason, such as Faraday rotation or different beam size effects. The remaining possibility is the intrinsic differences of the physical parameters involved in the maser process, such as the decay rate, Zeeman rate, maser size, and beaming angle.

3. The polarization angles of the 44 and $95 \mathrm{GHz}$ transition lines are well correlated in general, implying that both transitions are experiencing similar magnetic environments.
4. We did not observe any source with a fractional polarization $>30 \%$. Such high fractions were found by Wiesemeyer et al. (2004) and require anisotropic pumping/loss conditions. Our observations show that such conditions may be not as common in the starforming regions as Wiesemeyer et al. (2004) suggested.

5. We found that the polarization angles tend to be aligned perpendicular to the outflows for the seven maser sources with known outflow orientations. Generalizing this finding will require more samples. We also found that the maser polarization angles are not particularly correlated with the Galactic geometry.

6. We discussed some targets with peculiar polarization properties. Among them, DR21 $(\mathrm{OH})$ and $\mathrm{G} 82.58+0.20$ appear to be interesting targets because they show the $90^{\circ}$ polarization angle flip in the $44 \mathrm{GHz}$ polarization profiles, while it is not visible in their $95 \mathrm{GHz}$ polarization profiles. High angular-resolution polarimetry observations in both frequencies and future theoretical studies will reveal whether these polarization properties are due to the van Vleck angle crossing or due to a change in maser saturation level, providing more information on the methanol maser polarization physics.

We thank the anonymous referee whose suggestions have greatly improved the manuscript. We are grateful to all staff 
members in KVN who helped to operate the array and to correlate the data. The KVN is a facility operated by KASI (Korea Astronomy and Space Science Institute). The KVN operations are supported by KREONET (Korea Research Environment Open NETwork), which is managed and operated by KISTI (Korea Institute of Science and Technology Information). W.V. acknowledges support from ERC consolidator grant 614264. This research has made use of the SIMBAD database, operated at CDS, Strasbourg, France.

\section{REFERENCES}

Aumont, J., Conversi, L., Thum, C., et al. 2010, A\&A, 514, A70

Chapman, N. L., Davidson, J. A., Goldsmith, P. F., et al. 2013, ApJ, 770, 151 Crutcher, R. M. 2012, ARA\&A, 50, 29

Elitzur, M. 2002, in Astrophysical Spectropolarimetry, ed. J. Trujillo-Bueno, F. Moreno-Insertis, \& F. Sánchez (Cambridge: Cambridge Univ. Press), 225

Fernández-López, M., Girart, J. M., Curiel, S., et al. 2013, ApJ, 778, 72

Fish, V. L., \& Reid, M. J. 2006, ApJS, 164, 99

Garden, R. P., Hayashi, M., Hasegawa, T., Gatley, I., \& Kaifu, N. 1991, ApJ, 374,540

Goldreich, P., Keeley, D. A., \& Kwan, J. Y. 1973, ApJ, 179, 111

Gómez-Ruiz, A. I., Kurtz, S. E., Araya, E. D., Hofner, P., \& Loinard, L. 2016, ApJS, 222, 18

Han, J. L., Manchester, R. N., Lyne, A. G., Qiao, G. J., \& van Straten, W. 2006, ApJ, 642, 868

Han, J. L., \& Zhang, J. S. 2007, A\&A, 464, 609

Hull, C. L. H., Plambeck, R. L., Bolatto, A. D., et al. 2013, ApJ, 768, 159

Hull, C. L. H., Plambeck, R. L., Kwon, W., et al. 2014, ApJS, 213, 13

Kang, H., Kim, K.-T., Byun, D.-Y., Lee, S., \& Park, Y.-S. 2015, ApJS, 221, 6

Kang, S., Lee, S.-S., \& Byun, D.-Y. 2015, JKAS, 48, 257

Kelly, M. L., \& MacDonald, G. H. 1996, MNRAS, 282, 401

Kemball, A. J., \& Diamond, P. J. 1997, ApJL, 481, L111

Kim, K.-T., Byun, D.-Y., Bae, J.-H., et al. 2012, in IAU Symp. 287, Cosmic Masers - from $\mathrm{OH}$ to H0, ed. R. S. Booth et al. (Cambridge: Cambridge Univ. Press), 488

Kim, K.-T., Byun, D.-Y., Je, D.-H., et al. 2011, JKAS, 44, 81

Koch, P. M., Tang, Y.-W., \& Ho, P. T. P. 2010, ApJ, 721, 815

Kogan, L., \& Slysh, V. 1998, ApJ, 497, 800

Kurtz, S., Hofner, P., \& Álvarez, C. V. 2004, ApJS, 155, 149
Lee, S.-S., Byun, D.-Y., Oh, C. S., et al. 2011, PASP, 123, 1398

Mangum, J. G. 2000, Users Manusal for the NRAO 12 Meter Millimeter-Wave Telescope, NRAO, 129

Marseille, M., Bontemps, S., Herpin, F., van der Tak, F. F. S., \& Purcell, C. R. 2008, A\&A, 488, 579

Matsumoto, N., Hirota, T., Sugiyama, K., et al. 2014, ApJL, 789, 1

McIntosh, G. C., \& Predmore, C. R. 1993, ApJL, 404, L71

Menten, K. 1991, in ASP Conf. Ser. 16, Atoms, Ions and Molecules: New Results in Spectral Line Astrophysics, ed. A. D. Haschick \& P. T. P. Ho (San Francisco, CA: ASP), 119

Nedoluha, G. E., \& Watson, W. D. 1990, ApJ, 354, 660

Noutsos, A. 2012, SSRv, 166, 307

Oh, S.-J., Roh, D.-G., Wajima, K., et al. 2011, PASJ, 63, 1229

Pérez-Sánchez, A. F., \& Vlemmings, W. H. T. 2013, A\&A, 551, A15

Plambeck, R. L., \& Menten, K. M. 1990, ApJ, 364, 555

Sarma, A. P., \& Momjian, E. 2011, ApJL, 730, L5

Sault, R. J., Hamaker, J. P., \& Bregman, J. D. 1996, A\&AS, 117, 149

Schreyer, K., Stecklum, B., Linz, H., \& Henning, T. 2003, ApJ, 599, 335

Shi, H., Zhao, J.-H., \& Han, J. L. 2010, ApJL, 718, L181

Sridharan, T. K., Rao, R., Qiu, K., et al. 2014, ApJL, 783, L31

Stephens, I. W., Looney, L. W., Kwon, W., et al. 2014, Natur, 514, 597

Surcis, G., Vlemmings, W. H. T., Curiel, S., et al. 2011, A\&A, 527, A48

Surcis, G., Vlemmings, W. H. T., van Langevelde, H. J., \& Hutawarakorn Kramer, B. 2012, A\&A, 541, A47

Surcis, G., Vlemmings, W. H. T., van Langevelde, H. J., Hutawarakorn Kramer, B., \& Quiroga-Nuñez, L. H. 2013, A\&A, 556, A73

Tang, Y.-W., Ho, P. T. P., Koch, P. M., Guilloteau, S., \& Dutrey, A. 2013, ApJ, 763, 135

Val'tts, I. E., Ellingsen, S. P., Slysh, V. I., et al. 2000, MNRAS, 317, 315

Vlemmings, W. H. T. 2012, in IAU Symp. 287, Cosmic Masers - from OH to H0, ed. R. S. Booth et al. (Cambridge: Cambridge Univ. Press), 31

Vlemmings, W. H. T., \& Diamond, P. J. 2006, ApJL, 648, L59

Vlemmings, W. H. T., Surcis, G., Torstensson, K. J. E., \& van Langevelde, H. J. 2010, MNRAS, 404, 134

Wang, Y., Beuther, H., Bik, A., et al. 2011, A\&A, 527, A32

Wardle, J. F. C., \& Kronberg, P. P. 1974, ApJ, 194, 249

Wenger, M., Ochsenbein, F., Egret, D., et al. 2000, A\&AS, 143, 9

Wiesemeyer, H., Thum, C., \& Walmsley, C. M. 2004, A\&A, 428, 479

Williams, J. P., Plambeck, R. L., \& Heyer, M. H. 2003, ApJ, 591, 1025

Zhang, Q., Hunter, T. R., Brand, J., et al. 2005, ApJ, 625, 864

Zhang, Q., Qiu, K., Girart, J. M., et al. 2014, ApJ, 792, 116 